\title{
Wrinkling of Orthotropic Viscoelastic Membranes
}

\author{
X. Deng* and S. Pellegrino ${ }^{\dagger}$ \\ California Institute of Technology, Pasadena, California 91125 \\ DOI: 10.2514/1.J051255
}

\begin{abstract}
This paper presents a simplified simulation technique for orthotropic viscoelastic membranes. Wrinkling is detected by a combined stress-strain criterion and iterative scheme searches for the wrinkle angle using a pseudoelastic material stiffness matrix based on a nonlinear viscoelastic constitutive model. This simplified model has been implemented in ABAQUS/Explicit and is able to compute the behavior of a membrane structure by superposition of a small number of response increments. The model has been tested against a published solution for a time-independent isotropic membrane under simple shear and against experimental results on Stratofilm 420 under simple shear.
\end{abstract}

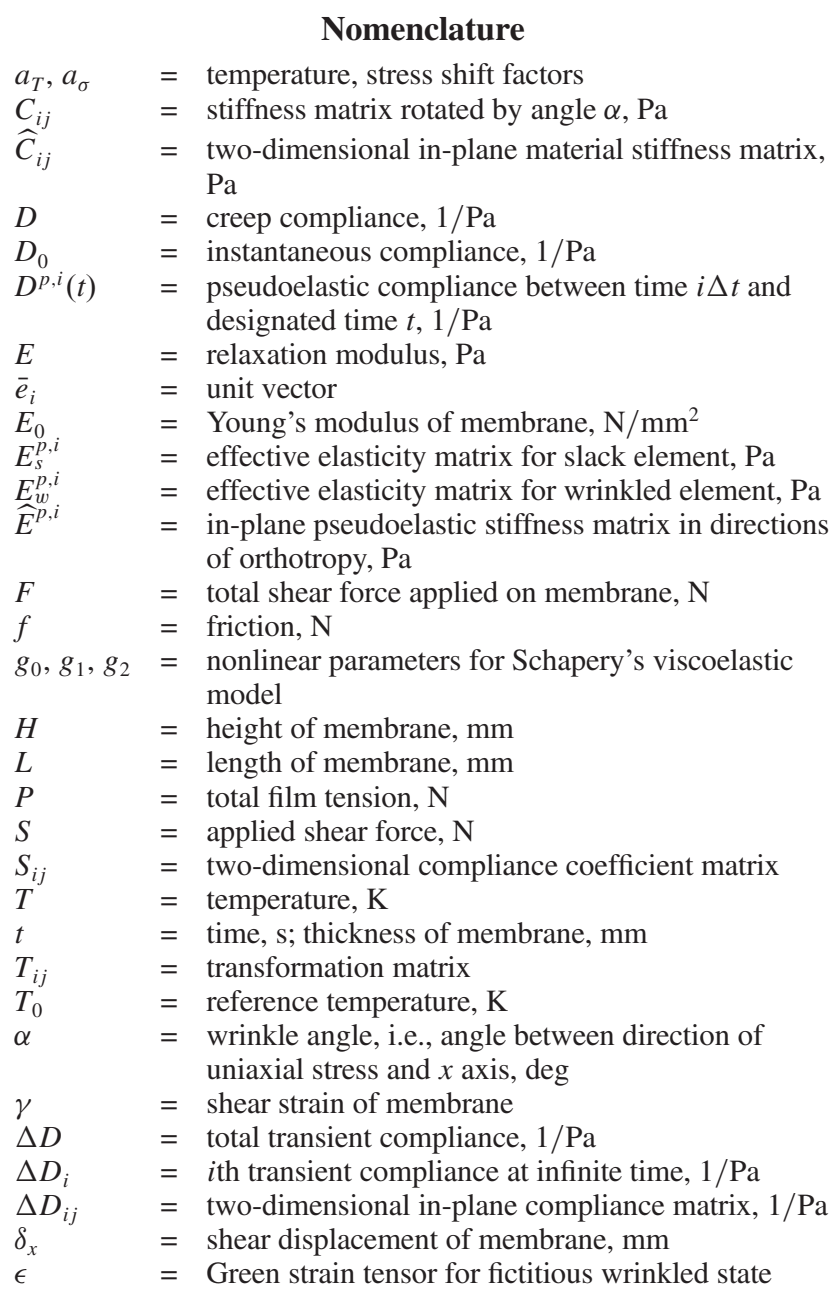

Presented as Paper 2010-2670 at the 11th AIAA Gossamer Forum, Orlando, FL, 12-15 April 2010; received 28 March 2011; revision received 23 June 2011; accepted for publication 14 July 2011. Copyright $(\odot 2011$ by Xiaowei Deng and Sergio Pellegrino. Published by the American Institute of Aeronautics and Astronautics, Inc., with permission. Copies of this paper may be made for personal or internal use, on condition that the copier pay the $\$ 10.00$ per-copy fee to the Copyright Clearance Center, Inc., 222 Rosewood Drive, Danvers, MA 01923; include the code 0001-1452/12 and \$10.00 in correspondence with the CCC.

*Graduate Student, Graduate Aerospace Laboratories, 1200 East California Boulevard.

${ }^{\dagger}$ Joyce and Kent Kresa Professor of Aeronautics and Professor of Civil Engineering, Graduate Aerospace Laboratories, 1200 East California Boulevard, MC 301-46; sergiop@ caltech.edu. Fellow AIAA.

$\begin{array}{lll}\epsilon^{u} & =\begin{array}{l}\text { Green strain tensor for state of natural uniaxial } \\ \text { tension }\end{array} \\ v & =\text { Poisson's ratio of membrane } \\ \rho & =\text { density of membrane, } \mathrm{kg} / \mathrm{mm}^{3} \\ \sigma_{\text {eff }} & =\text { effective stress, } \mathrm{Pa} \\ \tau_{i} & =i \text { th relaxation time, } \mathrm{s} \\ \psi & =\text { reduced time, } \mathrm{s}\end{array}$

\section{Introduction}

W RINKLING of thin membranes has attracted much interest, and yet little is known about wrinkles in anisotropic viscoelastic membranes. Our interest in this topic is motivated by current research in superpressure balloons, and particularly their behavior during inflation when the balloon envelope is heavily wrinkled. In recent papers [1,2], we have presented numerical simulations of some particular balloons that remain clefted when they are fully inflated and pressurized. These results were in agreement with experimental tests that had been conducted by the NASA Balloon Program, but the shape of the clefts in our simulations did not agree with the experimental observations. It was conjectured that these discrepancies may have resulted from the elastic and isotropic model for the balloon film that had been used. Hence, it was decided to implement a more detailed material model that would allow for both anisotropy and viscoelasticity. However, when wrinkling had been combined with these effects, we were unable to find any published test cases that could be used to confirm the validity of our simulations, and so we decided to carry out our own set of experiments as well.

Here, we present the outcome of our studies on wrinkling in moderately anisotropic, viscoelastic thin films. The paper begins with a literature review focused on a particular nonlinear viscoelastic material model for balloon film and an approach to the numerical simulation of wrinkling in anisotropic films. With this key background, in Sec. III, we present a computational scheme that models the viscoelasticity of the film with an incremental, pseudoelastic representation that is modified to allow for the formation of wrinkles when a combined stress-strain wrinkling criterion is satisfied. This scheme is then implemented in Sec. IV as a VUMAT subroutine in the finite element software ABAQUS/Explicit. Section V considers the special case of isotropic and elastic membranes, for which there is extensive literature, and compares the results from our simulations with some published results. Section VI presents a set of experiments that have been carried out on balloon film in a simple shear apparatus; the experimental setup and procedure are described, and the results of shear tests are presented. In Sec. VII, these experimental results are compared with the simulations. Section VIII concludes the paper.

\section{Background}

This section provides a compact review of viscoelasticity and wrinkling theories for orthotropic thin polymeric films. 


\section{A. Review of Viscoelasticity Theory}

In the classical theory of linear viscoelasticity [3,4], the creep compliance $D$ and relaxation modulus $E$ can be obtained from spring and dashpot models. A spring in series with a dashpot is known as the Maxwell model; a spring in parallel with a dashpot is known as the Kelvin-Voigt model. Both models have serious shortcomings. A better model is the standard linear solid model, which is either represented by a Maxwell element with an additional spring in parallel or a Kelvin-Voigt element with an additional spring in series.

A more realistic representation of actual viscoelastic behavior is obtained by considering $n$ Kelvin-Voigt elements with one spring in series, which leads to a compliance function expressed as a sum of exponential terms called the Prony series:

$$
D(t)=D_{0}+\sum_{i=1}^{n} \Delta D_{i}\left(1-e^{-t / \tau_{i}}\right)
$$

Viscoelastic materials are temperature- as well as time-dependent, but both effects can be combined through the time-temperature superposition principle by considering only the reference temperature $T_{0}$ and the reduced time:

$$
\psi(t) \equiv \int_{0}^{t} \frac{\mathrm{d} \tau}{a_{T}(T)}
$$

where $a_{T}(T)$ is the temperature shift factor, defined to be 1 at the reference temperature $\left[a_{T}\left(T_{0}\right)=1\right]$.

The compliance master curve, which defines the time-dependent compliance at the reference temperature, can be obtained from a series of uniaxial creep tests. It can be expressed as a combination of an instantaneous part $D_{0}$ and a transient part $\Delta D(\psi)$ :

$$
D(\psi)=D_{0}+\Delta D(\psi)
$$

Here, $D_{0}$ represents the instantaneous compliance at the reduced time $\psi=0$, and the transient part $\Delta D(\psi)$ can be represented by the Prony series:

$$
\Delta D(\psi)=\sum_{i=1}^{n} \Delta D_{i}\left(1-e^{-\psi / \tau_{i}}\right)
$$

The linear viscoelastic strain at time $t, \epsilon(t)$, is calculated by the convolution integral (also called the Boltzman superposition integral):

$$
\epsilon(t)=D_{0} \sigma(t)+\int_{0^{+}}^{t} \Delta D\left(\psi^{t}-\psi^{\tau}\right) \frac{\mathrm{d} \sigma(\tau)}{\mathrm{d} \tau} \mathrm{d} \tau \equiv D(\psi) \sigma(t)
$$

Experimental studies have shown that this linear model is acceptable at small strains; at larger strains, a nonlinear viscoelastic model has to be adopted, where the creep compliance is a function of stress, as well as of time and temperature. Following Schapery $[5,6]$, this behavior can be captured by introducing an experimentally derived stress-related shift $a_{\sigma}$ in the reduced time expression, which kicks in at stresses higher than a limiting value $\sigma_{0}$. Hence, Eq. (2) for the reduced time is rewritten as

$$
\psi(t) \equiv \int_{0}^{t} \frac{\mathrm{d} \tau}{a_{T}(T) a_{\sigma}(\sigma, T)}
$$

Schapery [5] has adopted a single-integral representation for the strain at time $t$ based on three nonlinear parameters $g_{0}, g_{1}$, and $g_{2}$ :

$$
\epsilon(t)=g_{0} D_{0} \sigma(t)+g_{1} \int_{0^{+}}^{t} \Delta D\left(\psi^{t}-\psi^{\tau}\right) \frac{\mathrm{d} g_{2} \sigma(\tau)}{\mathrm{d} \tau} \mathrm{d} \tau \equiv D(\psi) \sigma(t)
$$

where the nonlinear parameters are all equal to 1 in the linear viscoelasticity range.

The above uniaxial model has been extended [5] to plane stress loading of a polymeric film by making the assumption that the transient compliance in any direction can be expressed in terms of a constant matrix $S_{i j}$ multiplied by the compliance in a direction of orthotropy of the material, assumed to be known:

$$
\Delta D_{i j}=S_{i j} \Delta D_{11}
$$

where $S_{11}=1$ by definition. The uniaxial stress $\sigma$ used to calculate all stress-dependent nonlinearities in the previous model is then replaced with an effective stress. This is defined as

$$
\sigma_{\text {eff }}=\sqrt{\widehat{\sigma}_{11}^{2}+2 A_{12} \widehat{\sigma}_{11} \widehat{\sigma}_{22}+A_{22} \widehat{\sigma}_{22}^{2}+A_{66} \widehat{\tau}_{12}^{2}}
$$

where $\widehat{\sigma}_{11}$ is the normal stress in the one of the directions of material orthotropy, and it is assumed that the uniaxial model has been developed in this particular direction, $\widehat{\sigma}_{22}$ is the normal stress in the other direction of orthotropy, and $\widehat{\tau}_{12}$ is the shear stress.

\section{B. Wrinkling of Orthotropic Films}

There is extensive literature on wrinkling of thin elastic films. Key contributions were made by Stein and Hedgepeth [7], Miller and Hedgepeth [8], and Mansfield [9]; a comprehensive review can be found in Wong and Pellegrino [10-12]. Here, we shall focus specifically on wrinkling in orthotropic viscoelastic films, for which the literature is rather scarce.

Relevant previous work includes an analysis of wrinkling of viscoelastic membranes by Jenkins and Leonard [13] using a relaxed energy and dissipation function in a finite element analysis scheme. On the analytical front, a generalized tension field theory for anisotropic membranes with restricted types of nonlinear stressstrain relationships was proposed by Mansfield [14]. There is also a large amount of literature, starting from Reissner [15], in which artificial orthotropy was introduced as a numerical trick to derive compression-free solutions for isotropic membranes.

$\mathrm{Wu}[16,17]$ and $\mathrm{Wu}$ and Canfield [18] represented the true deformed surface of a wrinkled membrane by a smooth pseudodeformed surface, and hence introduced the wrinkle strain in this surface as a kinematic variable that measures the wrinkliness of the actual wrinkled surface. Based on the same basic ideas, Kang and Im [19] proposed an iterative scheme convenient for the finite element analysis of wrinkling in orthotropic membrane structures, which was later adopted by Gerngross and Pellegrino [20-22]. Alternative approaches have been proposed by Epstein and Forcinito [23] and Raible et al. [24].

The general idea in Kang and Im's paper [19] is that a wrinkled region in a thin film is in a state of uniaxial tension, and the orientation and magnitude of this tension can be obtained from an invariant relation between strain components. The actual wrinkled shape of the film is not of interest; instead, a fictitious nonwrinkled surface is used to describe the average deformation of the film.

Consider an undeformed material element $A B C D$ (Fig. 1a). Let $(\widehat{x}, \widehat{y})$ denote a Cartesian coordinate system in the undeformed configuration, where $\hat{x}, \hat{y}$ are aligned with the directions of orthotropy of the material. Let $(x, y)$ denote a rotated Cartesian coordinate system for the same material point but such that $x$ is the direction of the uniaxial stress in the wrinkled material. The rigidbody rotation angle $\alpha$ from $\widehat{x} \widehat{y}$ to $x y$ is called the wrinkling angle. It is noteworthy that for isotropic materials the wrinkling angle is the same as the principal stress or strain angle, but this is generally not the case for anisotropic materials.

After this rigid-body rotation, the material element, which is now considered in a rotated Cartesian coordinate system, is transformed from the undeformed configuration $A B C D$ to the final, wrinkled configuration $A^{\prime \prime} B^{\prime \prime} C^{\prime \prime} D^{\prime \prime}$ in two steps (Fig. 1b). The first step is a pure deformation from the undeformed configuration $A B C D$ to the deformed configuration $A^{\prime} B^{\prime} C^{\prime} D^{\prime}$, due to the application of the correct uniaxial stress $\sigma_{x}$. No wrinkling occurs during this deformation, because there are no constraints on the deformation kinematics. This deformation consists of a normal strain $\epsilon_{x}^{u}$, a transverse contraction $\epsilon_{y}^{u}$ (due to the Poisson's ratio of the membrane), and an additional shear strain $\gamma_{x y}^{u}$ (only in the case of anisotropic materials). This strain state is called the state of natural 


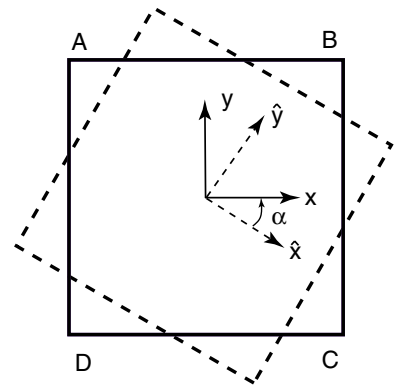

a) Rigid-body rotation of cartesian coordinate system

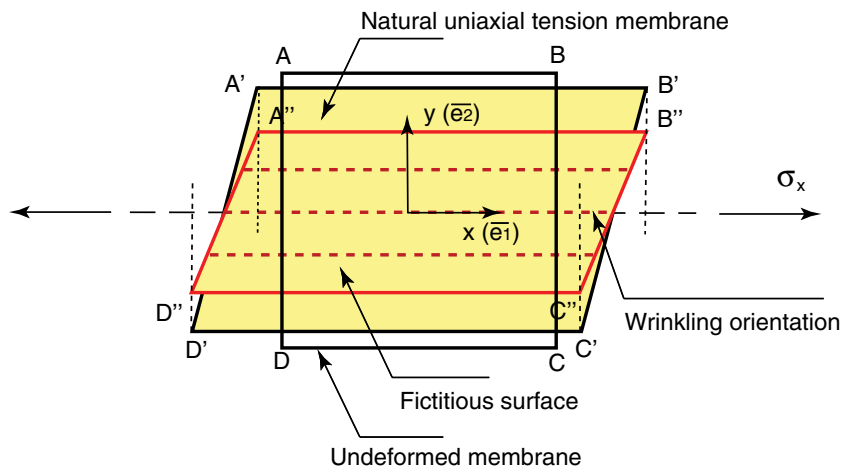

b) Deformation and wrinkling

Fig. 1 States of a wrinkled element.

uniaxial tension, and the superscript $u$ has been used to denote the corresponding strains. The second step involves pure wrinkling from $A^{\prime} B^{\prime} C^{\prime} D^{\prime}$ to $A^{\prime \prime} B^{\prime \prime} C^{\prime \prime} D^{\prime \prime}$. During this step, the stress in the material does not change; hence, its strain state also does not change, but the material element shrinks due to the formation of a series of wrinkles. At this point though, the actual material element (which is no longer planar) is replaced by a fictitious wrinkled surface with the same outer profile.

Let $\bar{e}_{1}$ and $\bar{e}_{2}$ be unit vectors denoting, respectively, the $x$ and $y$ directions. The material point $\bar{X}=X_{1} \bar{e}_{1}+X_{2} \bar{e}_{2}$ in the reference configuration $A B C D$ corresponds to point $\bar{x}=x_{1} \bar{e}_{1}+x_{2} \bar{e}_{2}$ in the state of natural uniaxial tension (i.e., in configuration $A^{\prime} B^{\prime} C^{\prime} D^{\prime}$ ) and to point $\bar{x}^{u}=x_{1}^{u} \bar{e}_{1}+x_{2}^{u} \bar{e}_{2}$ in the final deformed configuration $A^{\prime \prime} B^{\prime \prime} C^{\prime \prime} D^{\prime \prime}$. The mapping from $A B C D$ to $A^{\prime} B^{\prime} C^{\prime} D^{\prime}$ is written as $x_{1}^{u}=a^{u} X_{1}+c^{u} X_{2}$ and $x_{2}^{u}=b^{u} X_{2}$, and the mapping from $A B C D$ to $A^{\prime \prime} B^{\prime \prime} C^{\prime \prime} D^{\prime \prime}$ is written as $x_{1}=a X_{1}+c X_{2}$ and $x_{2}=b X_{2}$.

During the wrinkling process, the points $A^{\prime}, B^{\prime}, C^{\prime}$, and $D^{\prime}$ move vertically to $A^{\prime \prime}, B^{\prime \prime}, C^{\prime \prime}$, and $D^{\prime \prime}$; therefore, there is no deformation of $\bar{e}_{2}$, but there is still contraction of $\bar{e}_{1}$. That is,

$$
a=a^{u}, \quad c=c^{u} \text { and } \quad b \leq b^{u}
$$

The Green strain tensors for the fictitious wrinkled state and the state of natural uniaxial tension are

$$
\begin{aligned}
\epsilon= & \frac{1}{2}\left(a^{2}-1\right) \bar{e}_{1} \otimes \bar{e}_{1}+\frac{1}{2} a c\left(\bar{e}_{1} \otimes \bar{e}_{2}+\bar{e}_{2} \otimes \bar{e}_{1}\right) \\
& +\frac{1}{2}\left(b^{2}+c^{2}-1\right) \bar{e}_{2} \otimes \bar{e}_{2}=\epsilon_{x} \bar{e}_{1} \otimes \bar{e}_{1}+\epsilon_{x y} \bar{e}_{1} \otimes \bar{e}_{2} \\
& +\epsilon_{y x} \bar{e}_{2} \otimes \bar{e}_{1}+\epsilon_{y} \bar{e}_{2} \otimes \bar{e}_{2}
\end{aligned}
$$

$$
\begin{aligned}
\epsilon^{u} & =\frac{1}{2}\left[\left(a^{u}\right)^{2}-1\right] \bar{e}_{1} \otimes \bar{e}_{1}+\frac{1}{2}\left(a^{u}\right)\left(c^{u}\right)\left(\bar{e}_{1} \otimes \bar{e}_{2}+\bar{e}_{2} \otimes \bar{e}_{1}\right) \\
& +\frac{1}{2}\left[\left(b^{u}\right)^{2}+\left(c^{u}\right)^{2}-1\right] \bar{e}_{2} \otimes \bar{e}_{2}=\epsilon_{x}^{u} \bar{e}_{1} \otimes \bar{e}_{1}+\epsilon_{x y}^{u} \bar{e}_{1} \otimes \bar{e}_{2} \\
& +\epsilon_{y x}^{u} \bar{e}_{2} \otimes \bar{e}_{1}+\epsilon_{y}^{u} \bar{e}_{2} \otimes \bar{e}_{2}
\end{aligned}
$$

Comparing the above three equations, we conclude that, in the wrinkled state, the strain components $\epsilon_{x}$ and $\gamma_{x y}$ remain unchanged (i.e., $\epsilon_{x}=\epsilon_{x}^{u}$ and $\gamma_{x y}=\gamma_{x y}^{u}$ ), but the transverse strain $\epsilon_{y}$ is different due to the overcontraction of the material in the direction perpendicular to the stress direction (i.e., $\epsilon_{y}^{u} \geq \epsilon_{y}$ ).

Assuming that the Green strain $\epsilon$ in the membrane is small, it is approximately equal to the standard Cauchy strain, and so the stressstrain relationship has the standard linear form

$$
\left\{\begin{array}{c}
\widehat{\sigma}_{x} \\
\widehat{\sigma}_{y} \\
\widehat{\tau}_{x y}
\end{array}\right\}=\left[\begin{array}{ccc}
\widehat{C}_{11} & \widehat{C}_{12} & 0 \\
\widehat{C}_{21} & \widehat{C}_{22} & 0 \\
0 & 0 & \widehat{C}_{66}
\end{array}\right] \cdot\left\{\begin{array}{c}
\widehat{\epsilon}_{x} \\
\widehat{\epsilon}_{y} \\
\widehat{\gamma}_{x y}
\end{array}\right\}
$$

which can be written in the compact form $\{\widehat{\sigma}\}=[\widehat{C}]\{\widehat{\epsilon}\}$. Note that the shear-extension coupling terms in this material stiffness matrix $[\widehat{C}]$ are zero, because the relationship has been set up in the directions of orthotropy of the material. This stress-strain relationship can be transformed to the $x, y$ coordinate system by rotating through the angle $\alpha$ :

$$
\left\{\begin{array}{c}
\sigma_{x} \\
\sigma_{y} \\
\tau_{x y}
\end{array}\right\}=\left[\begin{array}{lll}
C_{11} & C_{12} & C_{16} \\
C_{21} & C_{22} & C_{26} \\
C_{61} & C_{62} & C_{66}
\end{array}\right] \cdot\left\{\begin{array}{c}
\epsilon_{x} \\
\epsilon_{y} \\
\gamma_{x y}
\end{array}\right\}
$$

or $\{\sigma\}=[C]\{\epsilon\}$. Here, $\{\epsilon\}$ can be obtained from $\{\widehat{\epsilon}\}$ by the transformation

$$
[C]=[T][\widehat{C}][R][T]^{-1}[R]^{-1}
$$

where $[R]=\operatorname{diag}\{1,1,2\}$, and

$$
[T]=\left[\begin{array}{ccc}
\cos ^{2}(\alpha) & \sin ^{2}(\alpha) & 2 \sin (\alpha) \cos (\alpha) \\
\sin ^{2}(\alpha) & \cos ^{2}(\alpha) & -2 \sin (\alpha) \cos (\alpha) \\
-\sin (\alpha) \cos (\alpha) & \sin (\alpha) \cos (\alpha) & \cos ^{2}(\alpha)-\sin ^{2}(\alpha)
\end{array}\right]
$$

The wrinkling angle $\alpha$ has to be such that the normal stress in the direction perpendicular to the wrinkles is zero and the shear stress is also zero; that is, $\sigma_{y}^{u}=\tau_{x y}^{u}=0$. If this condition is satisfied, then the transverse strain $\epsilon_{y}^{u}$ and the shear strain $\gamma_{x y}^{u}$ can be obtained from

$$
\begin{gathered}
\epsilon_{y}^{u}=\epsilon_{x}^{u} \frac{C_{21} C_{66}-C_{26} C_{61}}{C_{26} C_{62}-C_{22} C_{66}} \\
\gamma_{x y}^{u}=\epsilon_{x}^{u} \frac{C_{22} C_{61}-C_{21} C_{62}}{C_{26} C_{62}-C_{22} C_{66}}
\end{gathered}
$$

Kang and Im [19] proposed an iterative approach to search for the wrinkling angle; their procedure to find $\alpha$ is as follows. First, one determines a value of $\alpha$ that satisfies the relationship $\gamma_{x y}^{u}=\gamma_{x y}$, with $\epsilon_{x}^{u}=\epsilon_{x}$. Here, $\epsilon_{y}^{u}$ and $\gamma_{x y}^{u}$ are calculated from Eqs. (17) and (18). Finding $\alpha$ is equivalent to solving the equation

$$
f(\alpha)=\gamma_{x y}^{u}(\alpha)-\gamma_{x y}(\alpha)=0
$$

which may have multiple solutions between 0 and $180 \mathrm{deg}$. To find all of the solutions, we first divide the domain into 10 uniform intervals and look for a change of sign between two endpoints in any interval. If a change of sign is found, then there is at least one solution inside this particular interval. Then, we iteratively calculate the intersection between a straight line connecting the two endpoints and the $\alpha$ axis until the error is less than a prescribed tolerance. Once we have found a value of $\alpha$ that satisfies this equation, we check that $\epsilon_{y}^{u} \geq \epsilon_{y}$, and if this inequality is satisfied, then $\alpha$ defines the wrinkling orientation. If no change of sign is found, the domain is divided into 50 intervals and the calculation is repeated. 


\section{Effective Material Stiffness Matrix}

A particular balloon film, Stratofilm 420, will be considered in this study. This film has been extensively characterized by J. L. Rand. ${ }^{\ddagger}$ In the first part of this section, we review the nonlinear viscoelastic properties of Stratofilm 420 and then develop an approximate pseudoelastic constitutive model for a chosen temperature, time interval, and stress level; this approach was inspired by Stubstad and Simitses [25]. In the second part of the section, we modify the pseudoelastic coefficients to consider the effects of wrinkling.

\section{A. Pseudoelastic model for Stratofilm $\mathbf{4 2 0}$}

Stratofilm 420 is a $38-\mu$ m-thick film made of linear low-density polyethylene [26]. J. L. Rand (see footnote $\$$ ) has developed a nonlinear viscoelastic constitutive model for this film.

The transient compliance $\Delta D\left(T_{0}, \psi\right)$ in the machine direction of the film was given at a reference temperature $T_{0}=293.16 \mathrm{~K}$, and the instantaneous compliance $D_{0}=3.0 \times 10^{-10}\left(\mathrm{~Pa}^{-1}\right)$ was chosen to ensure that the transient compliance $D_{i}$ would all be positive. The coefficients of a 15-term Prony series are listed in Table 1.

The temperature shift factor $a_{T}$ was evaluated over the range of 163 to $323 \mathrm{~K}$ and then fitted at the reference temperature $T_{0}=293.16 \mathrm{~K}$. It is given by

$\log a_{T}$

$= \begin{cases}(T-293.16)\left[7.33 \times 10^{-4}(T-273.16)-0.179133\right] & T>233.16 \\ 3.1068-0.2350275(T-273.16) & T<233.16\end{cases}$

The stress shift was represented by $a_{\sigma}$ :

$$
\log a_{\sigma}=-0.126\left(\sigma_{\text {eff }}-\sigma_{0}\right)
$$

where $\sigma_{0}$ is the threshold at which linear behavior ends and nonlinear behavior begins:

$$
\sigma_{0}=69.527-0.430944 T+6.7962 \times 10^{-4} T^{2}
$$

Two of the nonlinear coefficients, $g_{0}$ and $g_{1}$, were set equal to 1 . The parameter $g_{2}$ was described by

$$
g_{2}=1+0.1875\left(\sigma_{\text {eff }}-\sigma_{0}\right)
$$

The coefficients for the biaxial model were, in addition to $S_{11}=1$,

$$
S_{22}=1.122+6.5895 \times 10^{-4} T-6.609 \times 10^{-6} T^{2}
$$

and $S_{12}=S_{21}=-0.58, S_{66}=4.45$.

The coefficients for the effective stress $A_{i j}$ were expressed as

$$
A_{22}=1.44, \quad A_{12}=-0.4 \quad \text { and } \quad A_{66}=0.8
$$

A finite element implementation of the above constitutive model for the analysis of a single balloon lobe has been developed by Gerngross and Pellegrino [20-22]. Here, we present an alternative approach that, although less accurate, is more suitable for large-scale simulations of balloon structures.

For a given time $t$, the integral representation for the strain $\epsilon(t)$ in Eq. (7) can be approximated by replacing the convolution integral with a series of $m$ equal substeps $\Delta t=\frac{t}{m}$, where each term is evaluated at the discrete time $t_{i}=i \Delta t$ :

$$
\begin{aligned}
\epsilon(t) \approx & g_{0} D_{0} \sigma(t)+g_{1} \sum_{i=1}^{m} \Delta D\left(\psi^{t}-\psi^{t_{i-1}}\right) \Delta\left[g_{2}\left(\sigma^{t_{i}}\right) \sigma\left(t_{i}\right)\right] \\
= & g_{0} D_{0} \sigma(t)+g_{1} \sum_{i=1}^{m} \Delta D\left(\psi^{t}-\psi^{t_{i-1}}\right)\left[g_{2}\left(\sigma^{t_{i}}\right) \sigma\left(t_{i}\right)\right. \\
& \left.-g_{2}\left(\sigma^{t_{i-1}}\right) \sigma\left(t_{i-1}\right)\right]
\end{aligned}
$$

Table 1 Prony series for Stratofilm SF420

\begin{tabular}{rcc}
\hline \hline$i$ & $D_{i}, \mathrm{MPa}^{-1}$ & $\tau_{i}, \mathrm{~s}$ \\
\hline 1 & $1.8764 \times 10^{-4}$ & $1.6548 \times 10^{-16}$ \\
2 & $2.9249 \times 10^{-5}$ & $4.8697 \times 10^{-15}$ \\
3 & $5.8224 \times 10^{-5}$ & $1.4330 \times 10^{-13}$ \\
4 & $8.7542 \times 10^{-5}$ & $4.2170 \times 10^{-12}$ \\
5 & $1.1561 \times 10^{-4}$ & $1.2409 \times 10^{-10}$ \\
6 & $1.4159 \times 10^{-4}$ & $3.6517 \times 10^{-9}$ \\
7 & $1.6989 \times 10^{-4}$ & $1.0746 \times 10^{-7}$ \\
8 & $2.0924 \times 10^{-4}$ & $3.1623 \times 10^{-6}$ \\
9 & $2.7274 \times 10^{-4}$ & $9.3057 \times 10^{-5}$ \\
10 & $3.7796 \times 10^{-4}$ & $2.7384 \times 10^{-3}$ \\
11 & $5.4670 \times 10^{-4}$ & $8.0582 \times 10^{-2}$ \\
12 & $8.0581 \times 10^{-4}$ & 2.3714 \\
13 & $1.1844 \times 10^{-3}$ & 69.783 \\
14 & $1.7204 \times 10^{-3}$ & 2053.5 \\
15 & $2.6285 \times 10^{-3}$ & 60,430 \\
\hline \hline
\end{tabular}

Substituting $g_{0}=g_{1}=1$ and assuming that $g_{2}$ changes slowly over $\Delta t$ (i.e., $\left.g_{2}\left(\sigma^{t_{i}}\right) \approx g_{2}\left(\sigma^{t_{i-1}}\right)\right)$, the above equation becomes

$$
\begin{aligned}
\epsilon(t) & \approx D_{0} \sum_{i=1}^{m} \Delta \sigma\left(t_{i}\right)+\sum_{i=1}^{m} g_{2}\left(\sigma^{t_{i}}\right) \Delta D\left(\psi^{t}-\psi^{t_{i-1}}\right) \Delta \sigma\left(t_{i}\right) \\
& =\sum_{i=1}^{m}\left[D_{0}+g_{2}\left(\sigma^{t_{i}}\right) \Delta D\left(\psi^{t}-\psi^{t_{i-1}}\right)\right] \Delta \sigma\left(t_{i}\right)
\end{aligned}
$$

Hence, we can define a pseudoelastic compliance $D^{p, i}$ (the superscript $p$ corresponds to pseudoelastic) between $i \Delta t$ and $t$ and consider the corresponding reduced times:

$$
D^{p, i}=D_{0}+g_{2} \Delta D\left(\psi^{t}-\psi^{t_{i-1}}\right)
$$

The corresponding strain can then be expressed as

$$
\epsilon(t)=\sum_{i=1}^{m} D^{p, i} \Delta \sigma\left(t_{i}\right)=\sum_{i=1}^{m} \Delta \epsilon\left(t_{i}\right)
$$

This uniaxial expression can be generalized to plane stress by defining the pseudoelastic compliance in the machine direction $\widehat{D}_{11}^{p, i}$ from Eq. (28):

$$
\widehat{D}_{11}^{p, i}=D_{0}+g_{2} \Delta D\left(\psi^{t}-\psi^{t_{i-1}}\right)
$$

and the remaining coefficients of the pseudoelastic compliance matrix are then

$$
\widehat{D}_{22}^{p, i}=S_{22} \widehat{D}_{11}^{p, i}, \quad \widehat{D}_{12}^{p, i}=\widehat{D}_{21}^{p, i}=S_{12} \widehat{D}_{11}^{p, i}, \quad \widehat{D}_{66}^{p, i}=S_{66} \widehat{D}_{11}^{p, i}
$$

It follows from Eq. (29) that the strain at time $t$ can be obtained as a sum of strain increments, which are calculated from the stress increments multiplied by the corresponding total pseudoelastic compliances. For each increment, the pseudoelastic compliance is constant.

For example, if we consider the conditions of constant temperature $T=293 \mathrm{~K}$ and constant stress $\widehat{\sigma}_{x}=4.5 \mathrm{MPa}, \widehat{\sigma}_{y}=5.0 \mathrm{MPa}$, and $\widehat{\tau}_{x y}=0 \mathrm{MPa}$, the shift of the compliance master curve in the machine direction is shown in Fig. 2. At time $t=1000 \mathrm{~s}$, the pseudoelastic moduli can be obtained from the compliance at the point marked with a dot in Fig. 2. They are, respectively, $\widehat{E}_{11}^{p}=90.98 \mathrm{MPa}$ and $\widehat{E}_{22}^{p}=121.68 \mathrm{MPa}$.

The pseudoelastic stiffness matrix in the directions of orthotropy of the film can be obtained by inversion of $\widehat{D}^{p, i}$ :

$$
\widehat{E}^{p, i}=\left[\begin{array}{ccc}
\widehat{E}_{11}^{p, i} & \widehat{E}_{12}^{p, i} & 0 \\
\widehat{E}_{21}^{p, i} & \widehat{E}_{22}^{p, i} & 0 \\
0 & 0 & \widehat{E}_{66}^{p, i}
\end{array}\right]
$$




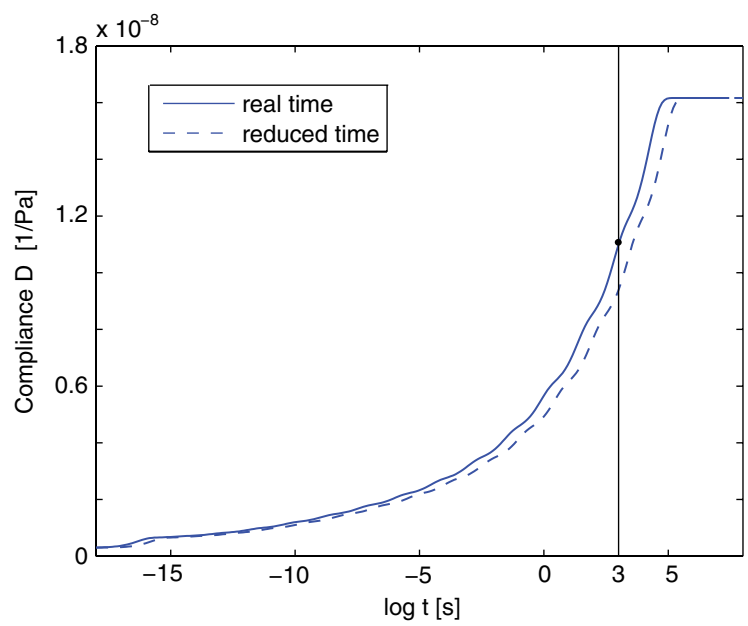

Fig. 2 Master curves of compliance in machine direction.

where

$$
\begin{aligned}
\widehat{E}_{11}^{p, i} & =\frac{1}{D_{11}^{p, i}} \frac{1}{S_{11}-S_{12}^{2} / S_{22}} \\
\widehat{E}_{12}^{p, i} & =E_{21}^{p, i}=\frac{1}{D_{11}^{p, i}} \frac{1}{S_{12}-S_{11} S_{22} / S_{12}} \quad \widehat{E}_{22}^{p, i}=\frac{1}{D_{11}^{p, i}} \frac{1}{S_{22}-S_{12}^{2} / S_{11}} \\
\widehat{E}_{66}^{p, i} & =\frac{1}{D_{11}^{p, i}} \frac{1}{S_{66}}
\end{aligned}
$$

\section{B. Correction for Wrinkling}

Kang and Im [19] compared three types of wrinkling criteria: the principal stress criterion, the principal strain criterion, and the mixed stress-strain criterion. They concluded that the mixed criterion works better for anisotropic wrinkling as it avoids numerical divergence caused by making wrong judgements about the membrane state, which happened with the principal stress-based criterion.

According to the mixed criterion, the effective elasticity matrix depends on the state of a membrane element:

Minor principal stress $\sigma_{2}>0$ : The membrane is taut, hence Eq. (32) is valid.

Minor principal stress $\sigma_{2} \leq 0$ and major principal strain $\varepsilon_{1}>0$ : The membrane is wrinkled.

Hence, we must solve for the wrinkle angle $\alpha$. First, we transform the pseudoelastic stiffness in Eq. (32) to the rotated coordinate system $x, y$ [the transformation is analogous to Eq. (14)]. In this system, the normal strain $\epsilon_{x}$ and the shear strain $\gamma_{x y}$ are equal to the strains in the state of natural uniaxial tension, whereas $\epsilon_{y}$ is not. The transverse stress $\sigma_{y}$ has to be zero in a coordinate system aligned with the wrinkles; hence,

$$
\sigma_{y}=E_{21}^{p, i} \epsilon_{x}+E_{22}^{p, i} \epsilon_{y}+E_{23}^{p, i} \gamma_{x y}=0
$$

From the above equation, $\epsilon_{y}$ can be expressed in terms of $\epsilon_{x}$ and $\gamma_{x y}:$

$$
\epsilon_{y}=-\frac{E_{21}^{p, i}}{E_{22}^{p, i}} \epsilon_{x}-\frac{E_{26}^{p, i}}{E_{22}^{p, i}} \gamma_{x y}
$$

Hence, we eliminate $\epsilon_{y}$ by substituting this expression into Eq. (14), and we obtain the effective elasticity matrix [22] for the wrinkled element:

$$
E_{w}^{p, i}=\left[\begin{array}{ccc}
E_{11}^{p, i}-\frac{E_{12}^{p, i} E_{21}^{p, i}}{E_{22}^{p, i}} & 0 & E_{13}^{p, i}-\frac{E_{12}^{p, i} E_{26}^{p, i}}{E_{22}^{p, i}} \\
0 & 0 & 0 \\
E_{61}^{p, i}-\frac{E_{62}^{p, i} E_{21}^{p, i}}{E_{22}^{p, i}} & 0 & E_{66}^{p, i}-\frac{E_{26}^{p, i} E_{62}^{p, i}}{E_{22}^{p, i}}
\end{array}\right]
$$

An alternative method is to directly use the strains in the state of natural uniaxial tension $\left\{\epsilon^{u}\right\}$ from Eqs. (17) and (18). Hence, the corrected stress in the wrinkled state $\{\sigma\}$ has to be a uniaxial tension, rewritten as $\{\sigma\}=\left\{\sigma^{u}\right\}=E^{p, i}\left\{\epsilon^{u}\right\}$. Since the element is under uniaxial tension, only the $x$ component $\sigma_{x}$ is nonzero. Finally, $\{\sigma\}$ is transformed back to $\{\widehat{\sigma}\}$ in the direction of orthotropy.

Major principal strain $\varepsilon_{1} \leq 0$ and minor principal stress $\sigma_{2}<0$ : The membrane is slack; hence, $E_{s}^{p, i}=[0]$.

In conclusion, to calculate the correct stress, the following steps are carried out:

Step 1: Check the state of the membrane element by means of the mixed criterion.

Step 2: If the element is wrinkled, find the wrinkle angle $\alpha$ from Kang and Im's scheme [19], described in Sec. II.

Step 3: Compute the corrected Cauchy stress using the effective elasticity matrix.

The stress and strain components used for the above wrinkling tests should ideally be the best current estimates of the values at time $t$. However, our current approach is to run each step $i$ as a separate simulation in ABAQUS/Explicit; hence, the only estimates that are available in each run are only the estimates based on the current increment.

\section{Finite Element Implementation}

In Sec. III, we have presented an approach to obtain approximate estimates of the stresses in a viscoelastic film at time $t$. The loading sequence is divided into $m$ steps, where $m$ is determined by the required precision, and

$$
\sigma(t)=\sum_{i=1}^{m} \Delta \sigma\left(t_{i}\right)=\sum_{i=1}^{m} \widehat{E}^{p, i} \Delta \epsilon\left(t_{i}\right)
$$

For example, the strain increments due to a three-step loading sequence are shown on the left of Fig. 3a; hence,

$$
\epsilon=\Delta \epsilon_{1}+\Delta \epsilon_{2}+\Delta \epsilon_{3}
$$

The stress at the end time $t_{3}$ due to the strain increments $\Delta \epsilon_{i}$ is computed with ABAQUS/Explicit in three separate analyses with compliances $\widehat{E}^{p, i}$. The results are shown in Figs. 3b-3d and are superposed in Fig. 3a. From the plots on the right in Fig. 3, the stress at time $t_{3}$ is given by

$$
\sigma\left(t_{3}\right)=\Delta \sigma_{1}+\Delta \sigma_{2}+\Delta \sigma_{2}=E^{p, 1} \Delta \epsilon_{1}+E^{p, 2} \Delta \epsilon_{2}+E^{p, 3} \Delta \epsilon_{3}
$$

Note that an approximation in this approach is to assume that the wrinkle directions in each step will not change significantly between the various steps. Also, the coefficient $g_{2}$ is stress-dependent and so is not correctly estimated for the full stress level. In other words, the proposed linear superposition is suitable for weakly nonlinear materials with only small wrinkle angle changes.

A user-defined material ABAQUS/Explicit subroutine (VUMAT) has been written to model wrinkling in an orthotropic viscoelastic film; it is schematically described in Fig. $4 .^{\S}$

\section{Verification for Isotropic Elastic Membranes}

We have tested our VUMAT implementation of wrinkling in ABAQUS/Explicit by means of a comparison with the analytical solution for a linear-elastic time-independent rectangular membrane under simple shear. The longer edges of the membrane are attached to rigid edges that are sheared uniformly, while the shorter edges are unconstrained. This problem was investigated by Wong and Pellegrino [12].

${ }^{\S}$ This subroutine can be downloaded from http://pellegrino.caltech.edu/ super_pressure_balloons.html [retrieved 2011]. 


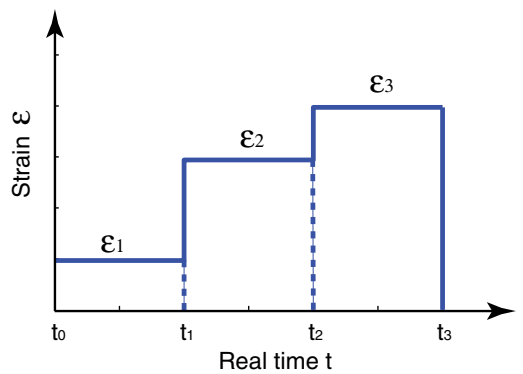

a)

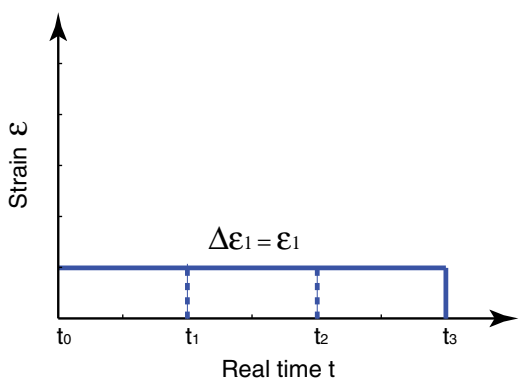

b)

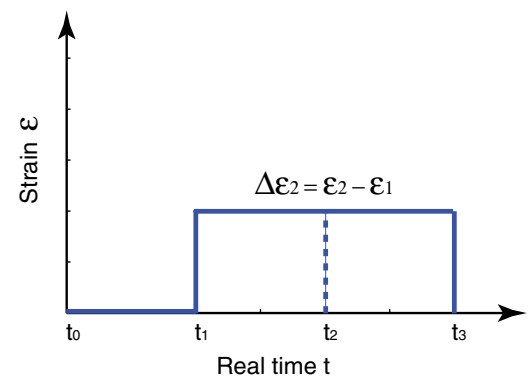

c)

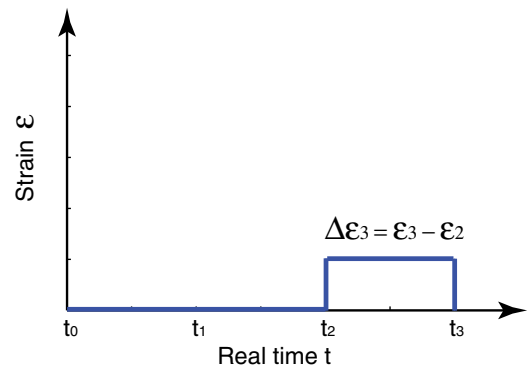

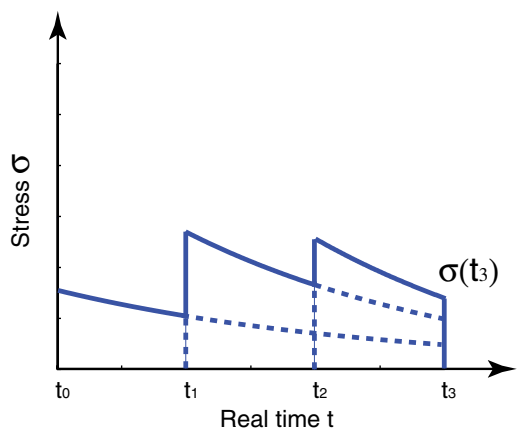
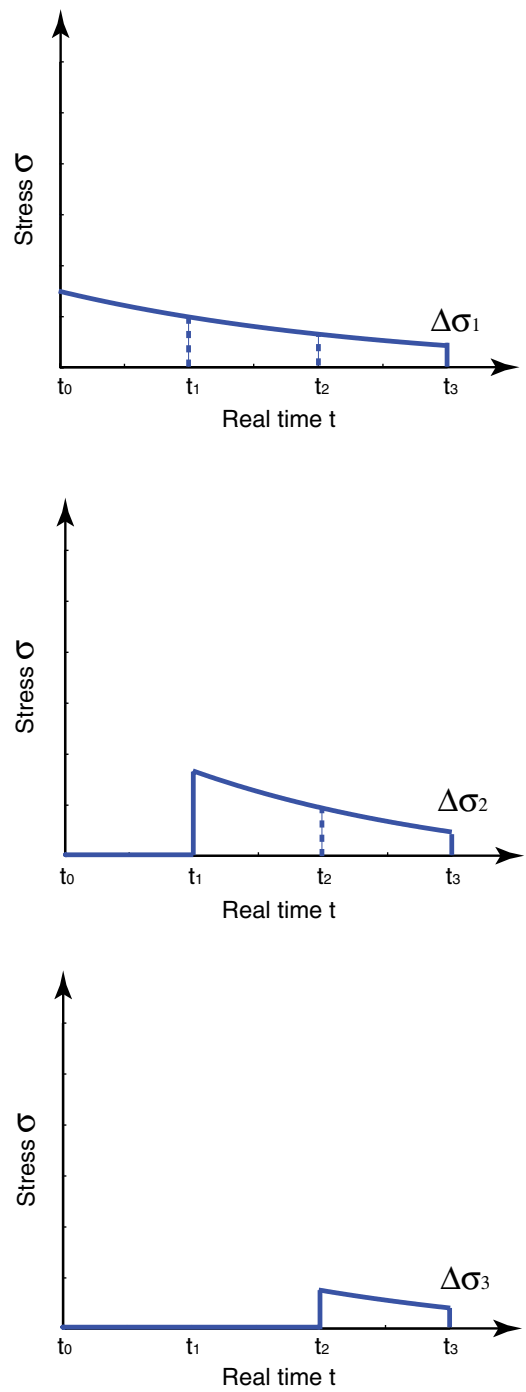

Fig. 3 Superposition of loading steps: a) total strain input and stress response, and b-d) incremental strain functions and corresponding stress responses.

\section{A. Construction of Finite Element Model}

This test case involves a rectangular membrane under simple shear [27]. The properties of the film used in the model and the geometrical dimensions of the rectangular membrane are summarized in Table 2.

The membrane was modeled with three-node fully integrated triangular membrane elements (M3D3). All translations of the bottom edge nodes were fully constrained, whereas the top edge nodes were left free. As shown in Fig. 5, the shear load was applied in terms of a horizontal shear displacement of the top edge.

The loading process consisted of two analysis steps: during the first step, lasting $2.5 \mathrm{~s}$, the upper edge nodes were moved by $3 \mathrm{~mm}$ in the $x$ direction, while all other translations were constrained. In the second step, also lasting $2.5 \mathrm{~s}$, all the translational degrees of freedom on the upper and lower edges were constrained to test the stability of the simulation.

\section{B. Results}

The stress distribution corresponding to the final value of the horizontal displacement of the membrane is illustrated by means of contour plots and a vector plot in Fig. 6. Since the major principal stress $\sigma_{1}$ is much greater than the minor principal stress $\sigma_{2}$, only the 


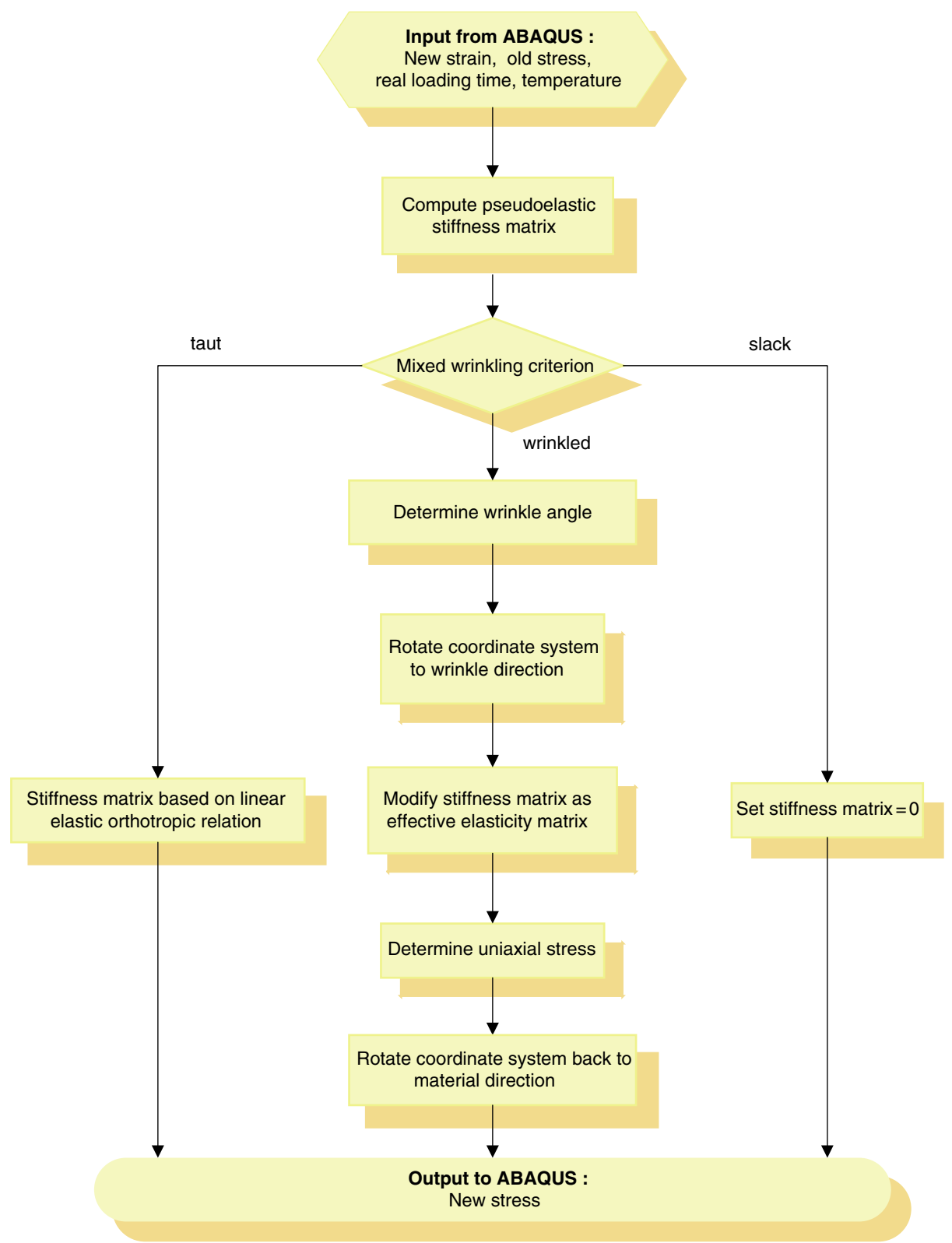

Fig. 4 Wrinkling algorithm for linear orthotropic viscoelastic material subroutine VUMAT,

major stress can be seen in the vector plot. The direction of the major principal stress, inclined at $45^{\circ}$, corresponds to the direction of the wrinkles. The dense distributions of vectors at the top right and bottom left corners indicate two areas of stress concentration.

The membrane finite element model using the VUMAT subroutine has succeeded in eliminating all negative stress, as illustrated by the minor stress distribution being approximately nonnegative everywhere.

The principal stresses across the middle of the membrane are plotted in Fig. 7. The principal stress $\sigma_{1}$ increases from zero at the edge to a uniform and positive value, $\sigma_{1}=41.36 \mathrm{MPa}$, whereas $\sigma_{2}$

Table 2 Summary of membrane properties

\begin{tabular}{lc}
\hline \hline Parameter & Value \\
\hline Length, $L, \mathrm{~mm}$ & 380 \\
Height, $H, \mathrm{~mm}$ & 128 \\
Thickness $t, \mathrm{~mm}$ & 0.025 \\
Young's modulus $E, \mathrm{~N} / \mathrm{mm}^{2}$ & 3530 \\
Poisson's ratio $v$ & 0.33 \\
Density $\rho, \mathrm{kg} / \mathrm{mm}^{3}$ & $1.46 \times 10^{-6}$ \\
\hline
\end{tabular}

remains approximately zero. The major principal strain is at $45^{\circ}$ and given by Wong and Pellegrino [11]:

$$
\epsilon_{1}=\frac{\gamma}{2}
$$

Since the shear strain is $\gamma=\delta_{x} / H, \sigma_{1}=E \epsilon_{1}=41.37 \mathrm{MPa}$. A detailed simulation with a thin-shell model of the membrane was carried out by Wong and Pellegrino [12], and the stress distribution obtained from that approach has been plotted for reference in Fig. 7. Figure 8 explains the wrinkling pattern for an isotropic membrane; it
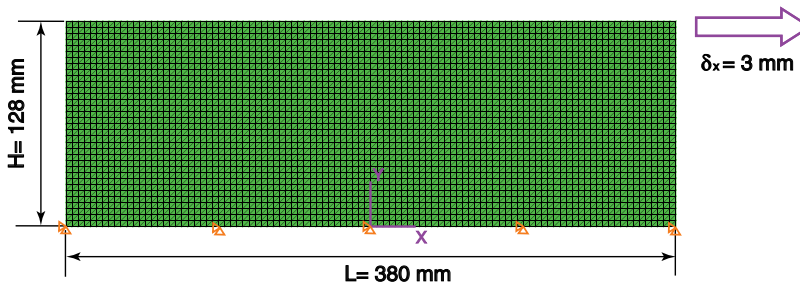

Fig. 5 Finite element mesh for membrane in shear. 

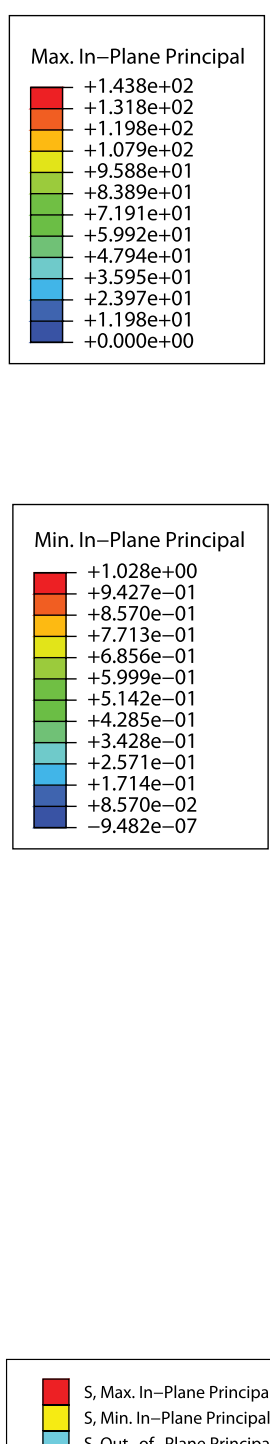

S, Max. In-Plane Principa $S$, Min. In-Plane Principal S, Out-of-Plane Principal

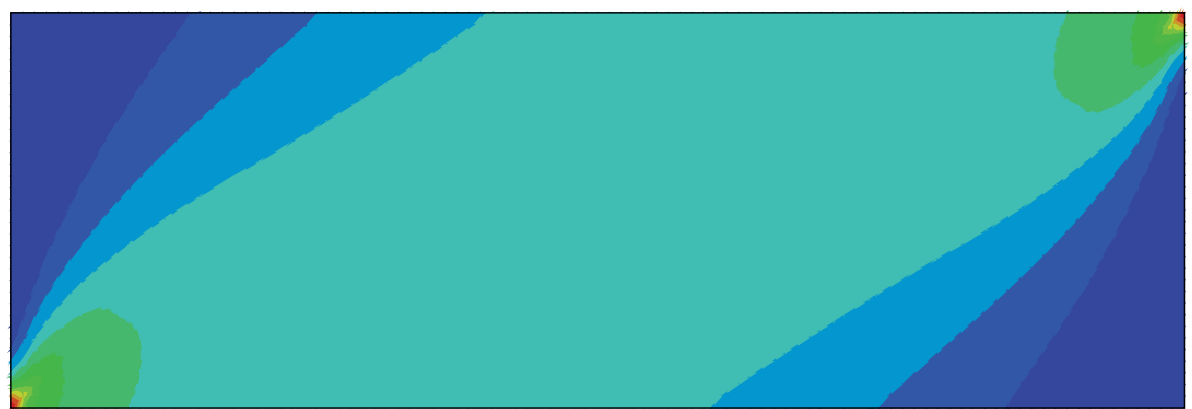

a) Major principal stress (unit: MPa)

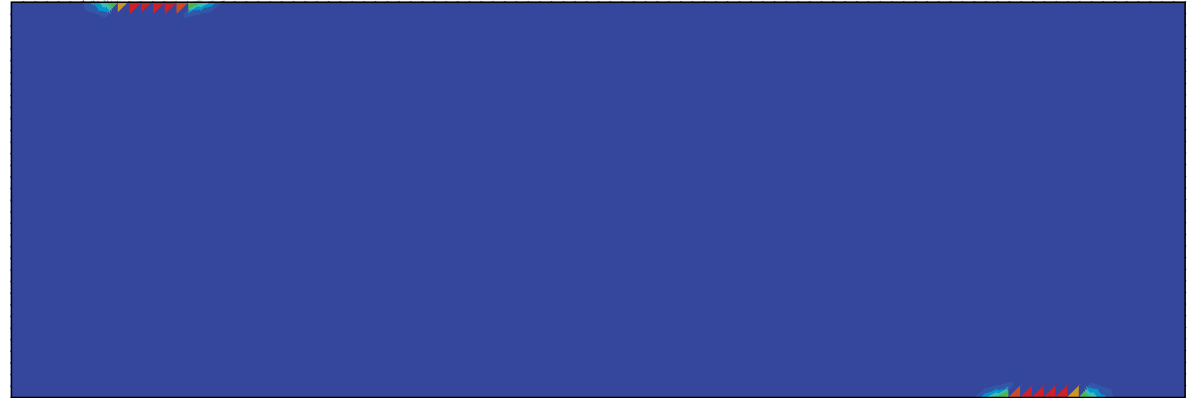

b) Minor principal stress (unit: MPa)

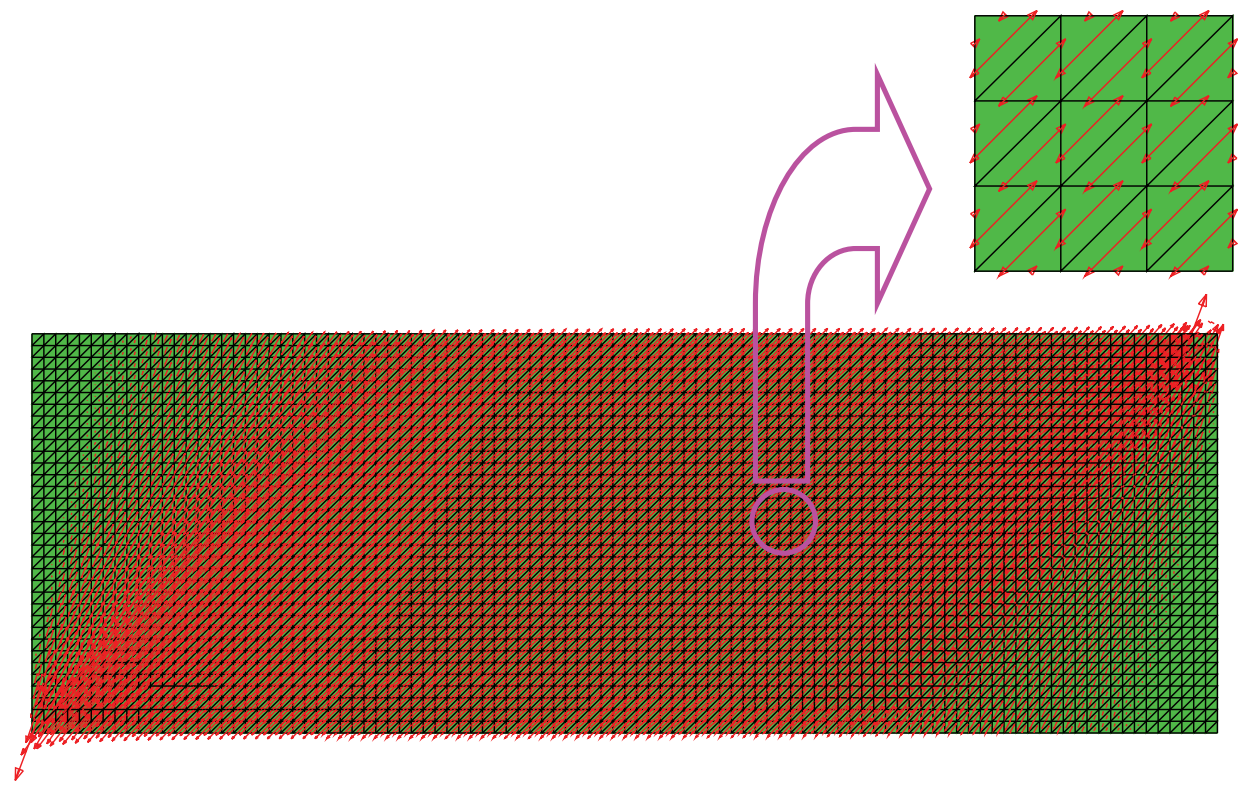

c) Vector plot

Fig. 6 Plot of principal stress distribution for isotropic time-independent membrane.

consists of a parallelogram region with a uniform wrinkling angle of $45^{\circ}$ plus two triangular regions that are slack. Note that there are stress concentrations at corners $\mathrm{B}$ and $\mathrm{D}$, whereas the stress is zero at the other two corners of the membrane, A and C.

The energy variation during the ABAQUS/Explicit simulation has been shown in Fig. 9. The viscous dissipation is negligible (the linear viscosity coefficient was set equal to 0.005 ), and the kinetic energy is also small, indicating that a quasi-static simulation has been achieved. A constant strain energy level of $236.76 \mathrm{~mJ}$ during the second step indicates that the simulation is stable.

\section{Experimental Studies of Viscoelastic Orthotropic Membranes}

A rectangular membrane with exactly the same dimensions as the one considered in Sec. V.A and subject to the same loading condition has been tested. However, now the situation is different in the following respects:

1) The membrane is Stratofilm 420, which is an orthotropic material showing nonlinear viscoelastic effects.

2) Both loading and unloading are measured, and hysteretic curves obtained from experiments are compared with results of pseudoelastic simulations.

3) After a linear ramp loading, the imposed displacement is kept constant for a certain time before unloading to observe the viscoelastic response.

\section{A. Shear Test Rig}

The scheme for the shear test was based on the concept previously adopted by Jenkins et al. [28] and then followed by Wong and Pellegrino [10]. The shear rig is shown in Fig. 10; note that the 


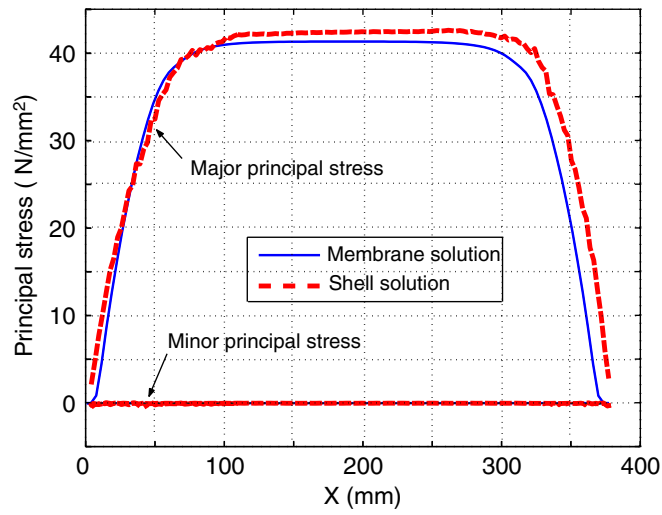

Fig. 7 Variation of principal stresses across middle horizontal section of isotropic time-independent membrane.

moving edge block is attached to four linear bearings (LUI 5AL). A key step in the preparation for the test is setting up the flat membrane surface without any visible imperfections. To do this, a mist of distilled water was sprayed on a flat Perspex sheet and a piece of Stratofilm 420 with the required dimensions was put on the sheet. Then, the film was lightly pressured with a hand roller to eliminate any air bubbles. The surface tension of the water at the interface holds the films against the plastic sheet. The upper and lower edges of one side of the film were glued to the fixed and moving edges of the rig, then clamping strips were glued and bolted to the other side of the film with M4 screws.

A controlled displacement of the moving edge block was imposed by means of a fine-threaded screw on the left-hand side of the rig, and a force sensor (Futek LCM300) coaxial with the screw was used to measure the compressive contact force $F$ between the screw and the moving edge block. A Keyence LK-G157 laser sensor placed on the right-hand side (not shown in the figure) was used to measure the displacement $\delta_{x}$, and the variation of force and displacement with time were measured with a Vishay System 7000 data logger. The scanning frequency was 128 samples per second during loading and unloading and 10 per minute when the displacement was held constant. The data samples were averaged using a window of 20 data points to reduce noise. The repeatability of the force sensor was $0.1 \%$ of full range, i.e., $\pm 1.11 \mathrm{~N}$; the resolution of the laser was $0.5 \mu \mathrm{m}$.

\section{B. Experimental Procedure}

Stratofilm 420 is orthotropic due to the nonuniform biaxial stretching during production. The directions of orthotropy are the machine direction (i.e., the direction in which the film is rolled) and the transverse direction. All experiments were carried out with the directions of orthotropy of the film parallel to the direction of shearing. In experiments denoted with an " $\mathrm{M}$ ", the machine direction of the film was parallel to the direction of shearing, whereas in experiments denoted with a "T", the transverse direction of the film was parallel to the direction of shearing. For each set of experiments, two displacement magnitudes were imposed, approximately 2 and $3 \mathrm{~mm}$; in the second set of experiments, the direction of motion was also reversed after holding the displacement constant for a time $t_{C}$. The test parameters are summarized in Table 3 .

It should be noted that, at some point during the reversed direction of motion, the reaction between the screw and the moving edge becomes tensile, and at this point, contact between the screw tip and the moving edge was lost. Hence, from this point on, the motion of the moving edge was governed by the condition that the reaction force should be zero.

\section{Correction for Friction}

The motion of the moving edge of the shear rig is resisted by friction in the linear bearings. It was assumed that the static and kinetic friction coefficients were equal and given by an expression of the type

$$
f=f_{0}+\mu P
$$

where $P$ is the total tension in the film, i.e., the total force in the direction perpendicular to the moving edge. The friction force $f$ was measured on the shear rig without the film by applying a known value

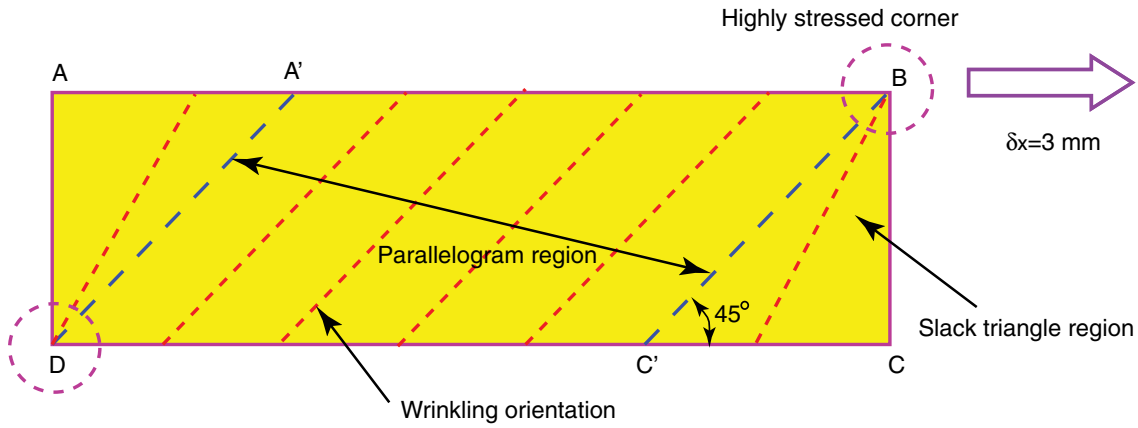

Fig. 8 Wrinkling pattern for isotropic time-independent membrane under simple shear.

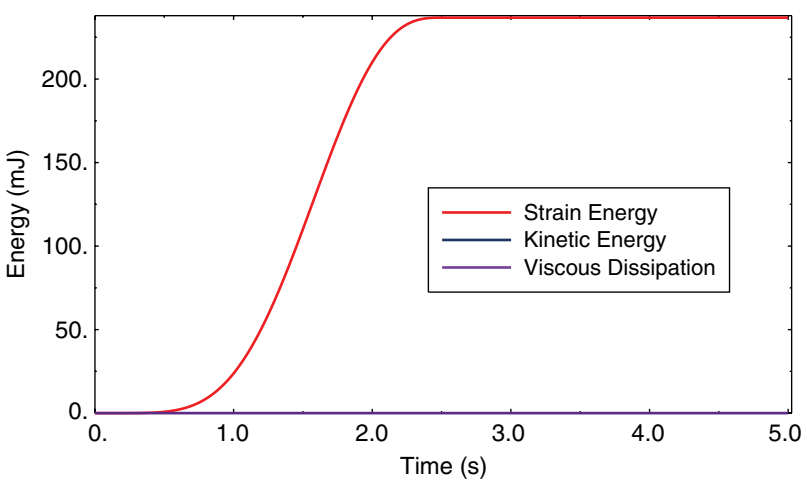

Fig. 9 Energy variation for isotropic time-independent membrane.

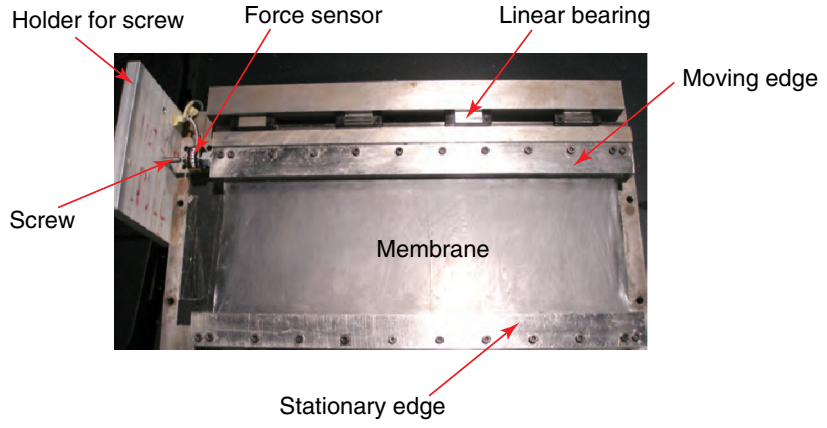

Fig. 10 Shear rig. 
Table 3 Test parameters ${ }^{\mathrm{a}}$

\begin{tabular}{lcccc}
\hline \hline & \multicolumn{3}{c}{ Test letter } \\
\cline { 2 - 5 } & $\mathrm{a}$ & $\mathrm{b}$ & $\mathrm{c}$ & $\mathrm{d}$ \\
\hline Direction of film & $\mathrm{M}$ & $\mathrm{M}$ & $\mathrm{T}$ \\
Displacement $\delta_{x}, \mathrm{~mm}$ & 2.31 & 3.36 & 2.39 & 2.34 \\
Temperature $T, \mathrm{~K}$ & 294.55 & 294.65 & 294.65 & Loading and unloading \\
Loading type & Loading only & Loading only & Loading and unloading & 16.79 \\
Linear ramp duration $t_{L}, \mathrm{~s}$ & 5.85 & 10.775 & 10.18 & $21,962.0$ \\
Constant displacement duration $t_{C}, \mathrm{~s}$ & $15,145.70$ & $13,582.83$ & $22,115.49$ & 5.578 \\
Linear ramp duration $t_{U}, \mathrm{~s}$ & N/A & N/A & 3.484 & \\
\hline \hline
\end{tabular}

${ }^{\mathrm{a} N} / \mathrm{A}$ denotes not applicable.

of $P$ and measuring the corresponding force needed to continuously move the sliding edge block.

The range of values of $P$ that was considered was based on an ABAQUS/Explicit simulation of the value corresponding to a film subject to a shear displacement $\delta_{x}=3 \mathrm{~mm}$. Hence, three levels of $P$ were applied: $0,11.121$, and $22.241 \mathrm{~N}$. Since the friction force $f$ has much smaller values than the horizontal force, a dynamic force sensor with higher precision, the PCB Piezotronics Model 208C01, was used to measure the friction force.

Two sets of test results have been plotted in Fig. 11. The linear fit, in newtons, is given by

$$
f=+1.2976+0.00421 P
$$

For simplicity, the constant value $f=1.298 \mathrm{~N}$ was assumed, as it turned out that $P$ was less than $22.241 \mathrm{~N}$ in all tests. In conclusion, the shear force acting on the film $S$ can be obtained by correcting the total applied force $F$ measured by the force sensor during the test; see Fig. 12 for details:

$$
\begin{aligned}
& S=F-f \quad \text { if } \dot{\delta}_{x}>0 \\
& S=F+f \quad \text { if } \dot{\delta}_{x}<0
\end{aligned}
$$

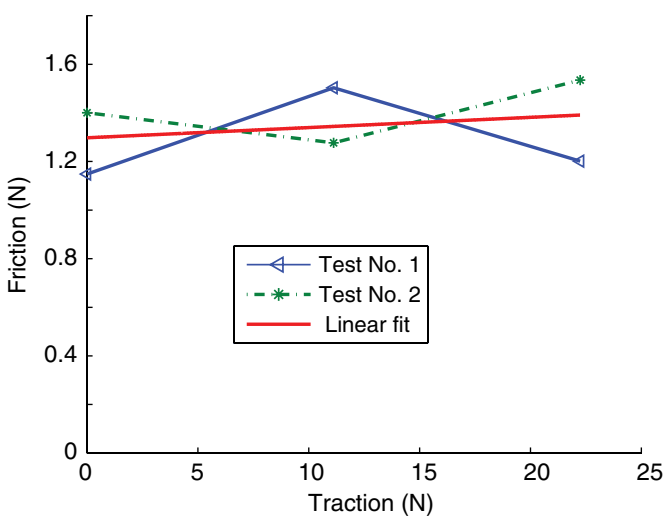

Fig. 11 Fitting of friction force.

\section{Results of Shear Tests}

The wrinkling pattern observed on a film subject to a $3 \mathrm{~mm}$ shear displacement is shown in Fig. 13. The wrinkles are almost evenly distributed in the central parallelogram region. The top left and bottom right corner regions are slack, and so there are no wrinkles in these regions.

A correction to the measured total force has been applied, using Eqs. (42) and (43) with a constant friction value, respectively, for the cases of loading and unloading. The results have been plotted in Figs. 14 and 15.

A comparison between the shear force time variation between the four tests is shown in Fig. 14. The increase in shear force is approximately linear during the loading ramp. Then, it gradually decreases when the displacement remains constant and tends to a constant value.

The relationship between the shear force and displacement for the four tests is shown in Fig. 15. The curves for total shear displacements of 2 and $3 \mathrm{~mm}$ generally follow the same shape, and kinks appear at the point of initial unloading due to the shear force correction when the friction suddenly changes direction. Clearly, the simplified friction correction model that has been used does not do a good job at the point of transition.

Since the differences in corresponding displacements are minor, the temperature did not change, and the loading can be assumed quasi static, it is interesting to compare the maximum shear forces in the

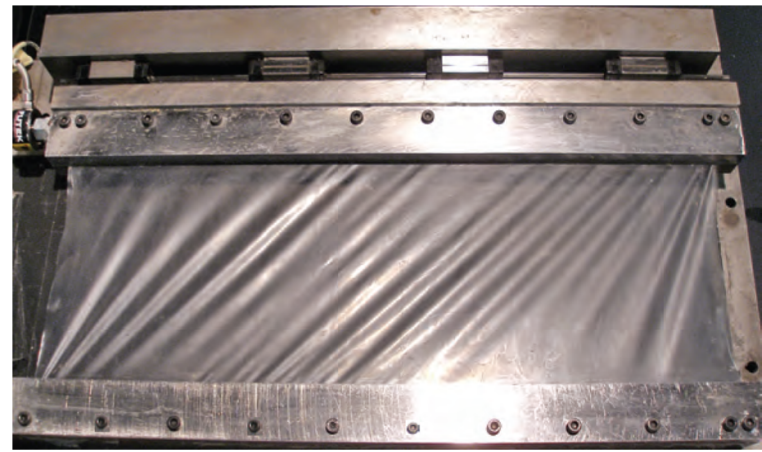

Fig. 13 Wrinkling pattern for $38-\mu$ m-thick Stratofilm 420 subject to $3 \mathrm{~mm}$ shear displacement.

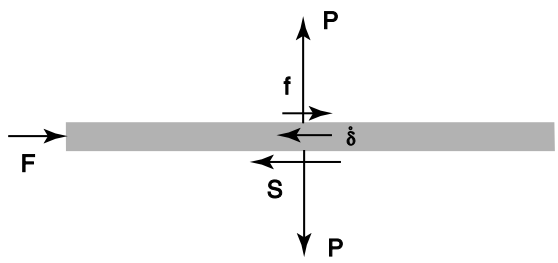

b)

Fig. 12 Free bodies of sliding edge block to derive correction of shear force. 


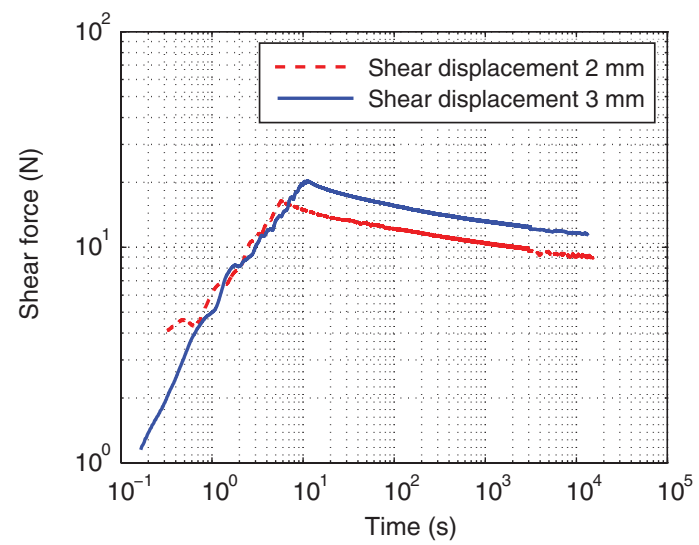

a)

Fig. 14 Measured time dependence of shear force, after correction for friction effects: a) machine direction parallel to direction of shearing, and b) transverse direction parallel to direction of shearing.

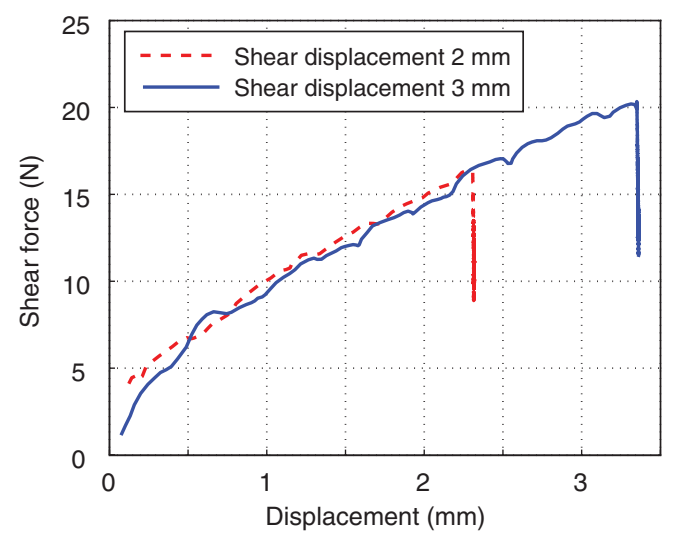

a)

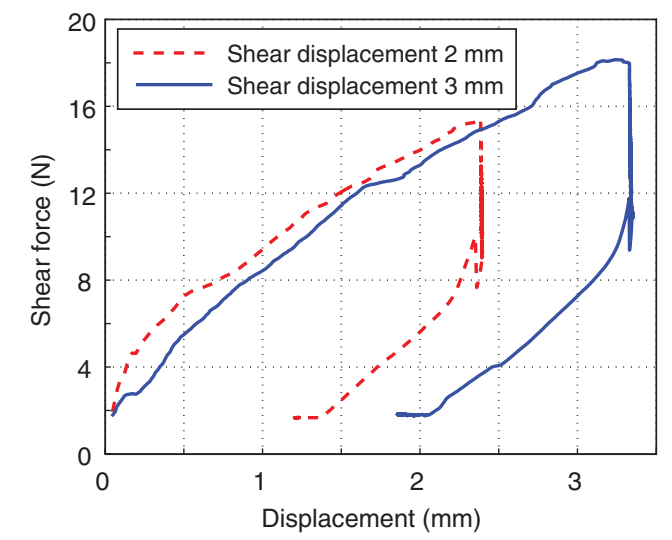

b)

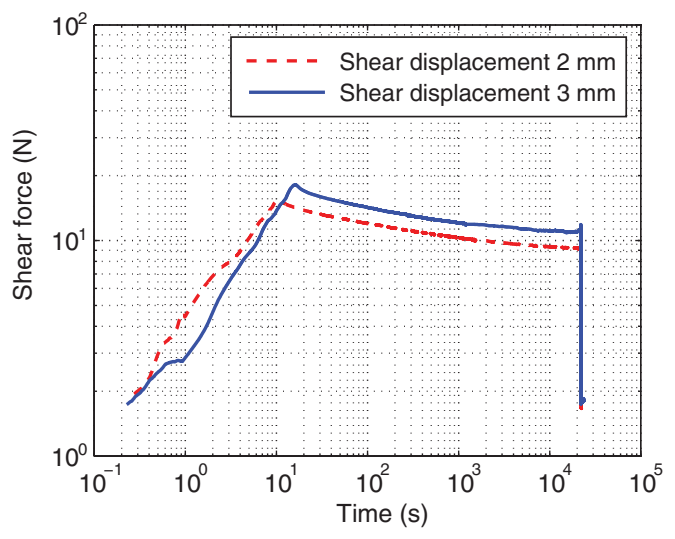

Fig. 15 Measured force-displacement relationships, after correction for friction effects: a) machine direction parallel to direction of shearing, and b) transverse direction parallel to direction of shearing.

four tests; see Table 4. With everything else fixed, one finds that the greater the shear displacement, the greater the shear force. Also, for the same displacement, the film arranged with the machine direction parallel to the long edge carries a higher shear force than the film placed in the transverse direction; however, the difference is generally less than $10 \%$. This is because Stratofilm 420 is only weakly anisotropic.

\section{Comparison of Results and Discussion}

The general orthotropic viscoelastic membrane model can be tested by comparing the simulation results to the results from the experiments. The first issue is how many steps should be used to represent the time history of the imposed displacement. As an example, we have investigated the linear loading ramp for the $\mathrm{M}$ test with a displacement $\delta_{x}=3.36 \mathrm{~mm}$ at the time $t_{L}=10.775 \mathrm{~s}$. We have found that the total estimate for the shear force at this time

Table 4 Maximum shear forces

\begin{tabular}{lcccc}
\hline \hline & \multicolumn{4}{c}{ Test letter } \\
\cline { 2 - 5 } & $\mathrm{a}$ & $\mathrm{b}$ & $\mathrm{c}$ & $\mathrm{d}$ \\
\hline Direction of film & $\mathrm{M}$ & $\mathrm{M}$ & $\mathrm{T}$ & $\mathrm{T}$ \\
Displacement $\delta_{x}, \mathrm{~mm}$ & 2.31 & 3.36 & 2.39 & 3.34 \\
Max total horizontal force $F, \mathrm{~N}$ & 17.618 & 21.206 & 16.633 & 19.271 \\
Max shear force $S, \mathrm{~N}$ & 16.320 & 19.908 & 15.335 & 17.973 \\
\hline \hline
\end{tabular}
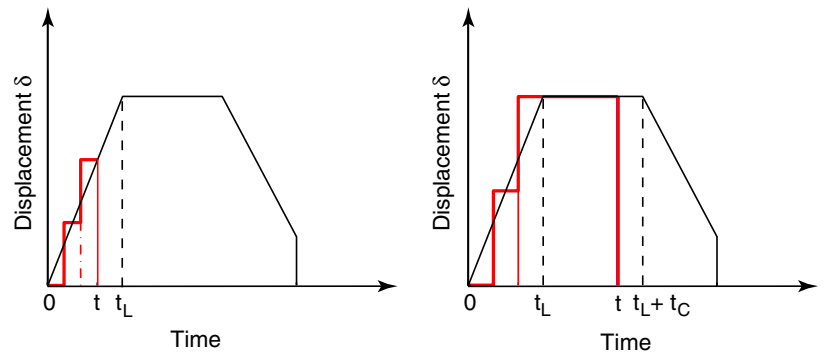

a)

b)

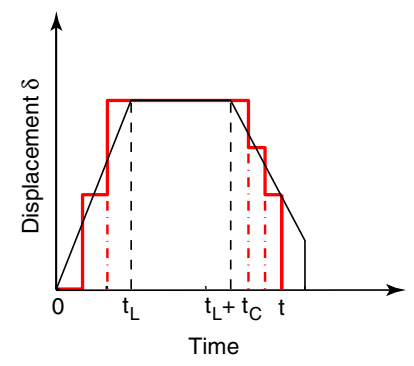

c)

Fig. 16 Discretization of displacement function using two steps for each ramp: a) case $t<t_{L}$, b) case $t_{L}<t<t_{L}+t_{C}$, and c) case $t_{L}+t_{C}<t<t_{L}+t_{C}+t_{U}$. 
increases from $16.445 \mathrm{~N}$ to 18.970 and $20.117 \mathrm{~N}$ for single-step, two-step, and three-step approximations, respectively. The first two results differ by $15 \%$, but the difference of the last two reduces to $6 \%$. Taking $20.117 \mathrm{~N}$ as the correct solution, we have assumed that a twostep approximation for linear ramp loading is sufficiently accurate. The experimental measurement of the force at this time was $19.908 \mathrm{~N}$, which confirms that the two-step solution is close to the physically correct value. At the stress-concentration corners of the membrane, there is a maximum principal strain of $5.2 \%$, which is at the limit of J. L. Rand's viscoelastic model (see footnote ${ }^{\ddagger}$ ).
Based on this observation, the discretization technique that has been adopted for the actual displacement function is shown in Fig. 16, depending on where the chosen time $t$ falls with respect to the ramp times.

The thing to note from the simulation results is that the wrinkle angles are on average 44.6 and $48.3^{\circ}$, respectively, in the $\mathrm{M}$ and $\mathrm{T}$ cases. This difference is too small to be detected in the experiments. Figures 17 and 18 compare the relationships between total shear force and time obtained from the simulations with both uncorrected and friction-corrected experimental results. During the initial

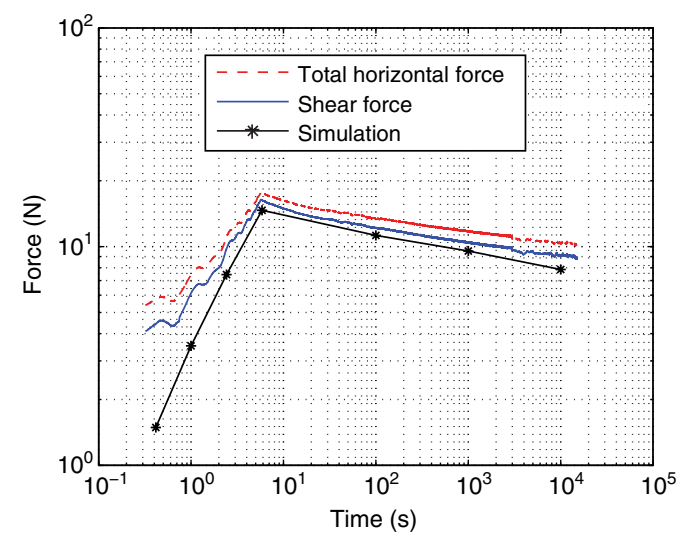

a)

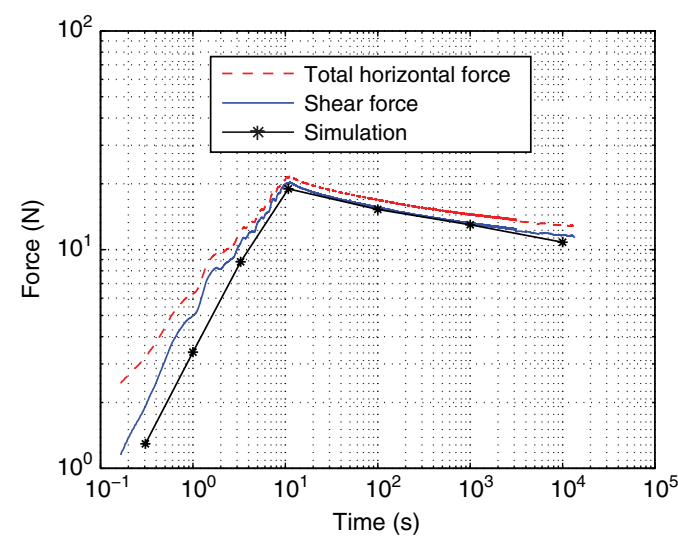

b)

Fig. 17 Comparison between simulation and measurement for $M$ test: a) maximum shear displacement 2 mm, and b) maximum shear displacement $3 \mathrm{~mm}$.

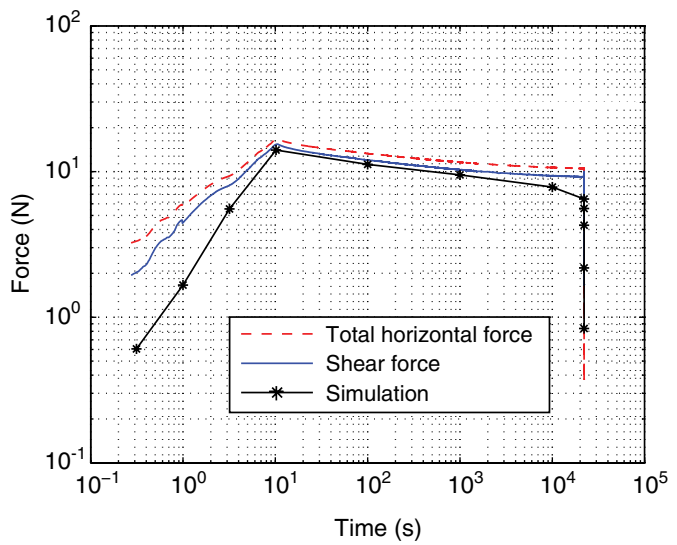

a)

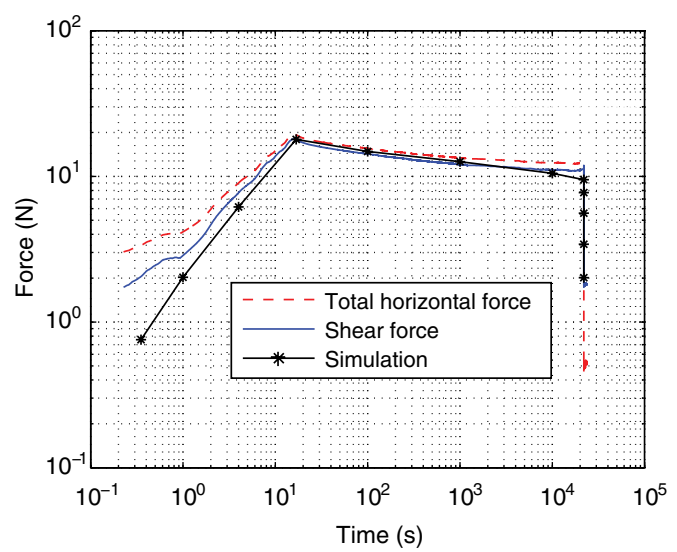

c)

Fig. 18 Comparison between simulation and experiment for $\mathbf{T}$ test: a) full behavior for maximum shear displacement 2 mm, b) unloading curve for maximum shear displacement $2 \mathrm{~mm}$, c) full behavior for maximum shear displacement 3 mm, and d) unloading curve for maximum shear displacement $3 \mathrm{~mm}$.

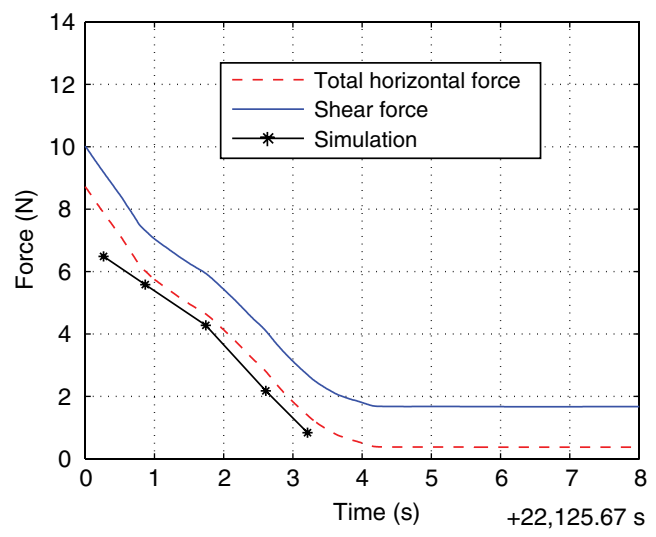

b)

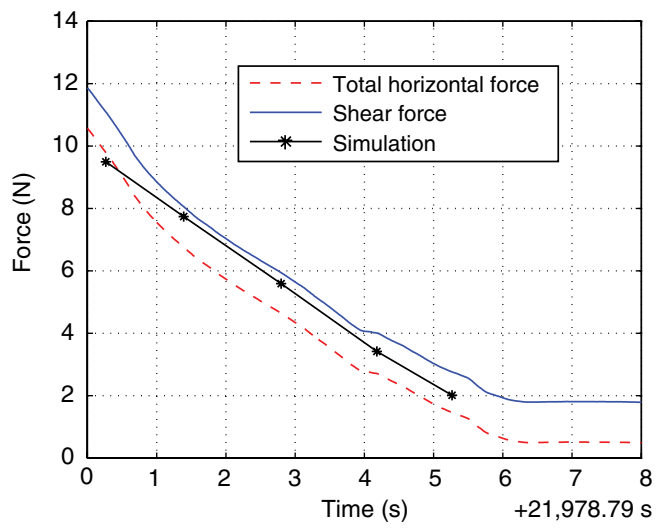

d) 


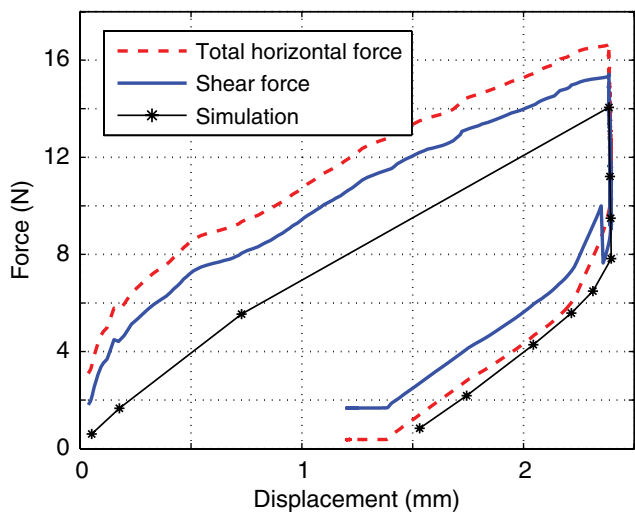

a)

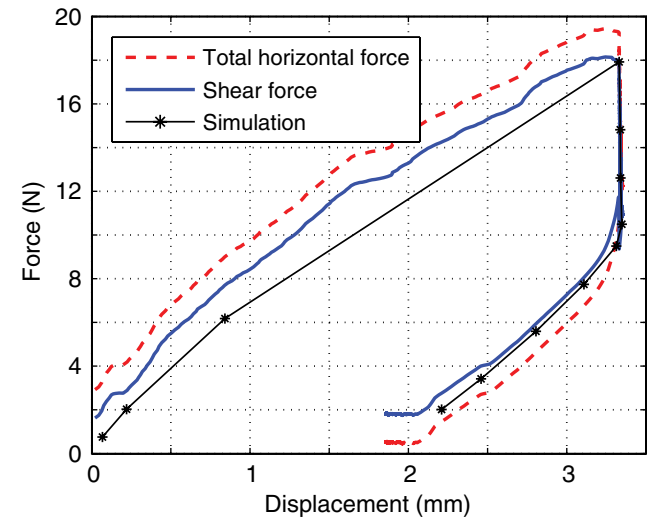

b)

Fig. 19 Comparison between simulation and experiment for $\mathrm{T}$ test: a) maximum shear displacement 2 mm, and b) maximum shear displacement 3 mm.

loading, especially the first $1 \mathrm{~s}$, the comparison shows significant differences; however, this is not surprising. The differences are due to the presence of inertia forces in the experimental results, due to the mass of the edge block that was not included in the finite element model, and to the fact that when the shear forces are small, the force sensor is less accurate and friction is comparatively large, and so our rather rough technique for removing friction effects is not accurate. The simulations and the experimental results are much closer from $t \approx 2 \mathrm{~s}$ onward, and particularly for the case $\delta_{x}=3 \mathrm{~mm}$. In Fig. 17b, during the constant displacement phase, the maximum difference between simulation and friction-corrected measured shear forces is $0.83 \mathrm{~N}$, or $7.15 \%$. In Fig. $18 \mathrm{c}$, the maximum difference is $0.58 \mathrm{~N}$, or $5.28 \%$. Figure $18 \mathrm{~d}$ provides a more detailed comparison of the unloading curves shown in Fig. 18c. In this range, the maximum difference is $0.74 \mathrm{~N}$.

The hysteretic relationship between shear force and displacement is shown in Fig. 19. Different loading and unloading paths result from the cumulative effects of viscoelasticity, which have a significant influence on the behavior of the wrinkled films.

\section{Conclusions}

The objective of this study was to investigate the wrinkling of orthotropic viscoelastic membranes. The method developed in this paper, based on a modification of the material stiffness matrix to incorporate the effects of orthotropic wrinkling and viscoelasticity, has been shown to be an effective way of capturing in a finite element simulation the behavior of a real polymer film.

In the wrinkling model, the state of a membrane element (taut, wrinkled, slack) is defined by a mixed criterion. Once it has been established that an element is wrinkled, an iterative scheme searches for the wrinkle orientation angle, and the correct stress distribution, involving only uniaxial tension in the wrinkle direction, is then obtained.

This wrinkling model has been validated by a comparison with a published solution for the case of a time-independent isotropic membrane under simple shear. The model has then been applied to a particular type of viscoelastic balloon film, known as Stratofilm 420. Using the Rand-Schapery model for this film, a time-dependent pseudoelastic stiffness matrix was defined, and so instead of having to compute the convolution integral throughout the simulation, the behavior of a membrane structure by superposition of a series of incremental response functions could be computed. These calculations were implemented in standard finite element software.

Experimental tests on Stratofilm 420 under simple shear were carried out, including loading and unloading, and the experimental results were compared with ABAQUS/Explicit simulations. The results agree very well once friction and dynamic effects are excluded. There are differences of about $10 \%$ in the shear forces measured on membranes arranged in different material directions because Stratofilm 420 has only weak anisotropy; hence, the direction of the wrinkles changed by less than $4^{\circ}$ when the film was rotated through $90^{\circ}$. Significant differences were seen between loading and unloading curves, indicating that viscoelastic behavior is a significant source of energy dissipation.

Viscoelastic simulations based on the convolution integral in Eq. (7) would be computationally intensive for large structures undergoing significant geometry changes, such as the deployment of balloons. Moreover, computing intermediate results at many stages of deployment of a balloon is of little interest, as the stresses remain small until the balloon reaches its final configuration and begins to pressurize. This paper has shown that, for the balloon film Stratofilm 420 , acceptable results are obtained from pseudoelastic simulations based on a two-step representation of each ramp in the loading function. Therefore, the application of the present approach to balloon simulations is expected to be more efficient than attempting to predict the detailed behavior of the balloon using a much finer representation of the loading together with an incremental form of the convolution integral.

\section{Acknowledgments}

This research was supported by the NASA Balloon Program Office (contract monitor Danny Ball). We thank the NASA SuperPressure Balloon Team for their contributions to this research. In particular, Loren Seely (Aerostar International) has provided film samples, and David Wakefield (Tensys Dynamics, Ltd.) and Jim Rand (Winzen Engineering, Inc.) have helped with valuable comments. This paper was selected as the Best Paper at the 11th AIAA Gossamer Systems Forum in Orlando, Florida.

\section{References}

[1] Deng, X., and Pellegrino, S., "Computation of Partially Inflated Shapes of Stratospheric Balloon Structures," 48th AIAA/ASME/ASCE/AHS/ ASC Structures, Structural Dynamics and Materials Conference, Schaumburg, IL, AIAA Paper 2008-2133, April 2008.

[2] Deng, X., and Pellegrino, S., "Finite Element Simulations of Clefting in Lobed Super-Pressure Balloons," AIAA Balloon Systems Conference, Seattle, WA, AIAA Paper 2009-2816, May 2009.

[3] Ward, I. M., Mechanical Properties of Solid Polymers, 2nd ed., Wiley, New York, 1985.

[4] McCrum, N. G., Buckley, C. P., and Bucknall, C. B., Principles of Polymer Engineering, 2nd ed., Oxford Science Publ., Oxford, England, U.K., 2003.

[5] Schapery, R. A., "On the Characterization of Nonlinear Viscoelastic Material," Polymer Engineering and Science, Vol. 9, No. 4, July 1969, pp. $295-310$ doi:10.1002/pen.760090410

[6] Schapery, R. A., "Nonlinear Viscoelastic and Viscoelastic Constitutive Equations Based on Thermodynamics," Mechanics of Time-Dependent Materials, Vol. 1, Dec. 1997, pp. 209-240. doi:10.1023/A:1009767812821

[7] Stein, M., and Hedgepeth, J. M., "Analysis of Partly Wrinkled Membranes," NASA Langley Research Center, TN D-813, 1961.

[8] Miller, R. K., and Hedgepeth, J. M., "An Algorithm for Finite Element Analysis of Partly Wrinkled Membranes," AIAA Journal, Vol. 20, 
No. 12,1982 , pp. $1761-1763$. doi: $10.2514 / 3.8018$

[9] Mansfield, E. H., The Bending and Stretching of Plates, 2nd ed., Cambridge Univ. Press, Cambridge, England, U.K., 1989.

[10] Wong, Y. W., and Pellegrino, S., "Wrinkled Membranes. Part I: Experiments," Journal of Mechanics of Materials and Structures, Vol. 1, No. 1, 2006, pp. 3-25. doi:10.2140/jomms.2006.1.3

[11] Wong, Y. W., and Pellegrino, S., "Wrinkled Membranes. Part II: Analytical Models," Journal of Mechanics of Materials and Structures, Vol. 1, 2006, pp. 27-61. doi:10.2140/jomms.2006.1.27

[12] Wong, Y. W., and Pellegrino, S., "Wrinkled Membranes. Part III: Numerical Simulations," Journal of Mechanics of Materials and Structures, Vol. 1, 2006, pp. 63-95. doi:10.2140/jomms.2006.1.63

[13] Jenkins, C. H., and Leonard, J. W., "Dynamic Wrinkling of Viscoelastic Membranes," Journal of Applied Mechanics, Vol. 60, 1993, Paper 575 doi:10.1115/1.2900841

[14] Mansfield, E. H., "Analysis of Wrinkled Membranes with Anisotropic and Non-Linear Elastic Properties," Proceedings of the Royal Society of London A, Vol. 353, 1977, pp. 475-498. doi:10.1098/rspa.1977.0046

[15] Reissner, E., "On Tension Field Theory," Proceedings Fifth International Congress of Applied Mechanics, 1938, pp. 88-92.

[16] Wu, C. H., "The Wrinkled Axisymmetric Air Bags Made of Inextensible Membranes," Journal of Applied Mechanics, Vol. 41, 1974, pp. 963-968. doi:10.1115/1.3423491

[17] Wu, C. H., "Nonlinear Wrinkling of Nonlinear Membranes of Revolution," Journal of Applied Mechanics, Vol. 45, 1978, pp. 533 538 doi:10.1115/1.3424357

[18] Wu, C. H., and Canfield, T. R., "Wrinkling in Finite Plane-Stress Theory," Quarterly of Applied Mathematics, Vol. 9, No. 1967, 1981, pp. $179-199$.

[19] Kang, S., and Im, S., "Finite Element Analysis of Wrinkling Membranes," Journal of Applied Mechanics, Vol. 64, June 1997, pp. 263-269.

doi:10.1115/1.2787302
[20] Gerngross, T., and Pellegrino, S., "Implementation and Validation of Schapery-Rand Anisotropic Viscoelasticity Model for Super-Pressure Balloons," AIAA Balloon Systems Conference, Williamsburg, VA, AIAA Paper 2007-2632, May 2007.

[21] Gerngross, T., and Pellegrino, S., "Modelling of Anisotropic Viscoelastic Behaviour in Super-Pressure Balloons," 48th AIAA/ ASME/ASCE/AHS/ASC Structures, Structural Dynamics and Materials Conference, Honolulu, HI, AIAA Paper 2007-1808, April 2007.

[22] Gerngross, T., and Pellegrino, S., "Anisotropic Viscoelasticity and Wrinkling of Superpressure Balloons: Simulation and Experimental Verification," AIAA Balloon Systems Conference, Seattle, WA, AIAA Paper 2009-2815May 2009.

[23] Epstein, M., and Forcinito, M. A., "Anisotropic Membrane Wrinkling: Theory and Analysis," International Journal of Solids and Structures, Vol. 38, 2001, pp. 5253-5272. doi:10.1016/S0020-7683(00)00346-2

[24] Raible, T., Tegeler, K., Lohnert, S., and Wriggers, P., "Development of a Wrinkling Algorithm for Orthotropic Membrane Materials," Computer Methods in Applied Mechanics and Engineering, Vol. 194, 2005, pp. 2550-2568. doi:10.1016/j.cma.2004.07.045

[25] Stubstad, J. M., and Simitses, G. J., "Bounding Solutions of Geometrically Nonlinear Viscoelastic Problems," AIAA Journal, Vol. 24, No. 11, 1986, pp. 1843-1850. doi: $10.2514 / 3.9533$

[26] Rainwater, E. L., and Smith, M. S., "Ultra High Altitude Balloons for Medium-to-Large Payloads," Advances in Space Research, Vol. 33, No. 10, 2004, pp. 1648-1652. doi: 10.1016/j.asr.2003.07.037

[27] Ogden, R. W., Non-Linear Elastic Deformations, Dover, Minneola, NY 1997, p. 101

[28] Jenkins, C. H., Haugen, F., and Spicher, W. H., "Experimental Measurement of Wrinkling in Membranes Undergoing Planar Deformation," Experimental Mechanics, Vol. 38, 1998, pp. 147-152. doi:10.1007/BF02321658
M. Hyer Associate Editor 